\title{
Reference approach in theory of pseudospin systems
}

\author{
R.R.Levitskii, S.I.Sorokov, O.R.Baran \\ Institute for Condensed Matter Physics \\ of the National Academy of Sciences of Ukraine, \\ 1 Svientsitskii Str., 79011 Lviv, Ukraine
}

Received April 18, 2000

For theoretical description of pseudospin systems with essential shortrange and long-range interactions we use the method based on calculations of the free energy functional taking into account the short-range interactions within the reference approach in cluster approximation. We propose a consistent formulation of the cluster expansion method for quantum pseudospin systems. We develop a method allowing one to obtain within the cluster approximation an Ornstein-Zernike type equation for reference cumulant Green function of an arbitrary order. In the two-particle cluster approximation we derived an explicit expression for pair temperature cumulant Green function of the reference system. In the cluster random phase approximation we calculated and studied thermodynamic characteristics, elementary excitation spectrum, and integral intensities of the Ising model in transverse field.

Key words: phase transitions, pseudospin models, reference approach, cluster approximation, Ornstein-Zernike equation, soft mode

PACS: 03.65.-w, 05.30.-d

\section{Introduction}

Modern statistical theory of condensed media pays a great attention to the studies of ferroelectric and magnetic materials, described by pseudospin models with essential short-range and a long-range interactions, especially of hydrogen-bonded ferroelectrics [1-12] and low-dimensional magnets [13,14]. For an adequate description of these objects, such an approach is required that would allow to use different techniques to take into account a short-range and a long-range interaction. This is a typical mathematical problem in theories of multiparticle systems. It has been successfully solved in the studies of equilibrium properties of classical systems [1521] and metals [21-25] using the approach proposed in [15-18,22,24,25]. Within this approach, the long-range and short-range interactions are described in phase spaces of collective variables and individual coordinates, respectively. The system with short-range interaction is called then the reference system. 
Using the idea of separating the reference system [15-18], in [26-30] a method was proposed for description of pseudospin systems with essential short-range and long-range interactions. This method is based on the calculation of the free energy functional taking into account the short-range interactions within the reference approach. In $[26,27,30]$ expansions of the free energy functional and functionals of the temperature cumulant Green functions (CGF) in the inverse long-range interaction radius were studied. For the first time there has been performed a total summation of the reducible in blocks diagrams in the free energy functional and of non-compact diagrams in functionals of CGFs for quantum pseudospin models. Expressions for the free energy and temperature CGFs of the considered systems were obtained. It was shown how to obtain consistent approximations for their thermodynamic and dynamic characteristics, using classification of the approximations for free energy functional according to loop diagrams.

It should be noted that the general expressions for thermodynamic and dynamic characteristics of pseudospin systems with short-range and long-range interactions obtained in [26-30] contain thermodynamic and correlation functions of the reference system. Hence, to solve a general problem one needs to solve a reference one, that is, to calculate free energy and CGFs of the reference system. The maximal order of the reference CGFs depends on the order of the approximation for the longrange interactions. Depending on the reference Hamiltonian, the reference problem can be solved exactly (see, for instance, [14,31-36]) or approximately, taking into account peculiarities of the reference system. The best description of the reference system for a wide class of pseudospin models can be obtained based on the cluster expansions method (see [1-12,37-39]). In some papers [40-43] this method was successfully used to study disordered magnetic and ferroelectric materials. Unfortunately, the cluster method was correctly developed only for Hamiltonians with commuting single-particle (describing the interaction of pseudospins with external and internal fields) and multiparticle parts (describing the interaction between pseudospins). It was mostly used to calculate thermodynamic characteristics of pseudospin models. In [44-46] a problem of calculating the distribution functions for Ising models within the cluster approach was considered but not solved completely. Equations for pair correlation functions of the reference system (Ornstein-Zernike type equation) in $[44,45]$ were not derived consistently but constructed artificially. The problem of calculating the quasimomentum-dependent pair correlation functions was also considered in [46] within the cluster approach. The results obtained are valid in paraphase only. Later, a method was proposed $[47,48]$, which allows one to obtain Ornstein-Zernike type equations for arbitrary order correlation functions of Ising models. These equations for pair and three-particle correlation functions were derived and solved within the two-particle cluster approximation (TPCA). It was shown that the cluster approach to the calculation of correlation functions of the reference Ising models proposed in $[47,48]$ yields the known exact results $[31,32]$ for pair and three-particle correlation functions of the one-dimensional Ising model.

In $[48,49]$, using the four-particle cluster approximation, pair $\vec{q}$-dependent correlation functions of deuterons were calculated for $\mathrm{KD}_{2} \mathrm{PO}_{4}$ type ferroelectrics and 
$\mathrm{ND}_{4} \mathrm{D}_{2} \mathrm{PO}_{4}$ type antiferroelectrics. Dynamics of hydrogen-bonded ferroelectrics taking into account the tunnelling effects was considered in [50-52] within the original approach proposed in [50]. For the first time it has been shown, that in the reference approach, with the short-range interactions and tunnelling taken into account in cluster approximation, the dynamic properties of the studied systems are to a great extent determined by an effective tunnelling parameter, renormalized by the short-range interactions. Later, this peculiarity of the dynamic properties of hydrogen-bonded compounds was also noticed in [53]. Unfortunately, expressions for dynamic (at $\vec{q}=0$ and $E=0$ ) and static characteristics, calculated in [50-52], turned out to be inconsistent. That results from the fact, that dynamic characteristics were obtained using the method of two-time temperature Green functions, equations for which were decoupled in the spirit of Tyablikov approximation. Thus, the intracluster Green functions of the reference system were connected only via the long-range interactions, whereas the short-range correlations were not taken into account. Thus, the method has not been developed, which would allow one to consistently describe thermodynamic and dynamic characteristics of reference quantum pseudospin models.

In the present paper, for a theoretical description of pseudospin systems with essential short-range and long-range interactions we shall use the self-consistent reference approach developed in [26-30]. In section 2 we shall briefly consider the main results obtained within this approach. Then, a consistent formulation of cluster expansion method for reference quantum pseudospin systems will be given for the first time. We shall propose a method, allowing to obtain Ornstein-Zernike type equations for reference temperature cumulant Green functions of an arbitrary order within a cluster approximation. An Ornstein-Zernike type equation for the pair correlator will be derived and solved in the two-particle cluster approximation. The last section is devoted to the investigation of the Ising model in transverse field (IMTF) within the cluster random phase approximation (CRPA) using the results obtained in this paper.

\section{Theory of pseudospin system with short-range interactions taken into account in reference approach}

We consider pseudospin systems with short-range and long-range interactions, described by the Hamiltonian

$$
\mathcal{H}(\{\Gamma\})=-\beta H=\sum_{\nu=1}^{N} \sum_{a} \Gamma_{\nu}^{a} S_{\nu}^{a}+\frac{1}{2} \sum_{\nu, \delta} \sum_{a, b} K^{a b} S_{\nu}^{a} S_{\nu+\delta}^{b}+\frac{1}{2} \sum_{\nu, \mu} \sum_{a, b} J_{\nu \mu}^{a b} S_{\nu}^{a} S_{\mu}^{b}
$$

Here $K^{a b}$ and $J_{\nu \mu}^{a b}$ are the short-range and the long-range parts of the pair interactions. $S_{\nu}^{a}(a=x, y, z$ or,,$+- z)$ are components of a normalized $\left(S^{z}=-1,1\right)$ spin $\vec{S} . \mathcal{H}(\{\Gamma\})$ means $\mathcal{H}(\{\Gamma\})=\mathcal{H}\left(\Gamma_{1}^{a_{1}}, \ldots, \Gamma_{N}^{a_{1}}, \Gamma_{1}^{a_{2}}, \ldots, \Gamma_{N}^{a_{2}}, \Gamma_{1}^{a_{3}}, \ldots, \Gamma_{N}^{a_{3}},\right)$. Hereafter, the argument $\{\Gamma\}$ will be frequently omitted. The factor $\beta=1 /\left(k_{\mathrm{B}} T\right)$, occurring in 
$\Gamma, K$, and $J$ (in the above presented form of the Hamiltonian $H$ ), will be written explicitly only in some of the final expressions.

After an identity transformation of the operators $S_{\nu}^{a}=\left\langle S_{\nu}^{a}\right\rangle+\Delta S_{\nu}^{a}$ in the last term of Hamiltonian (2.1), which describes the long-range interactions between pseudospins, we obtain

$$
\mathcal{H}(\{\Gamma\})={ }^{k} \mathcal{H}(\{\varkappa\})-\frac{1}{2} \sum_{\nu, \mu} \sum_{a, b} J_{\nu \mu}^{a b}\left\langle S_{\nu}^{a}\right\rangle\left\langle S_{\mu}^{b}\right\rangle+\frac{1}{2} \sum_{\nu, \mu} \sum_{a, b} J_{\nu \mu}^{a b} \Delta S_{\nu}^{a} \cdot \Delta S_{\mu}^{b} .
$$

The first term in (2.2) describes the short-range interactions between pseudospins placed in a field created by the long-range interactions and by $\Gamma_{\nu}^{a}$

$$
\begin{aligned}
& { }^{k} \mathcal{H}(\{\varkappa\})=-\beta \cdot{ }^{k} H(\{\varkappa\})=\sum_{\nu=1}^{N} \sum_{a} \varkappa_{\nu}^{a} S_{\nu}^{a}+\frac{1}{2} \sum_{\nu, \delta} \sum_{a, b} K^{a b} S_{\nu}^{a} S_{\nu+\delta}^{b} ; \\
& \varkappa_{\nu}^{a}=\Gamma_{\nu}^{a}+\sum_{\mu=1}^{N} \sum_{b} J_{\nu \mu}^{a b}\left\langle S_{\mu}^{b}\right\rangle .
\end{aligned}
$$

The Hamiltonian ${ }^{k} H(\{\varkappa\})$ is called the reference Hamiltonian [26]. The argument $\{\varkappa\}$ will be often dropped. Let us note that in the mean field approximation (MFA) over the long-range interactions, the last term in (2.2) is neglected.

Our main task is to calculate the free energy

$$
F(\{\Gamma\})=-k_{\mathrm{B}} T \ln Z(\{\Gamma\}), \quad Z(\{\Gamma\})=\mathrm{Sp} \mathrm{e}^{\mathcal{H}}
$$

and pair temperature cumulant Green functions

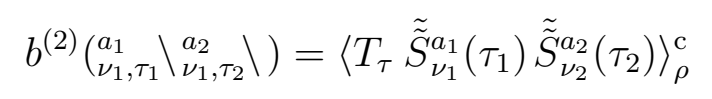

for the models, described by Hamiltonian (2.1). Here

$$
\tilde{\tilde{S}}_{\nu}^{a}(\tau)=\mathrm{e}^{-\tau \mathcal{H}} S_{\nu}^{a} \mathrm{e}^{\tau \mathcal{H}}
$$

the averaging is performed with the density matrix

$$
\rho=\rho(\{\Gamma\})=[Z(\{\Gamma\})]^{-1} \cdot \mathrm{e}^{\mathcal{H}} .
$$

For the sake of convenience, in our calculations we do not use the free energy $F(\{\Gamma\})$ but the $\mathcal{F}(\{\Gamma\})$-function (logarithm of the partition function).

According to the theory proposed in [26], to solve the formulated problem, one should calculate the ${ }^{k} \mathcal{F}$-function

$$
{ }^{k} \mathcal{F}(\{\varkappa\})=\ln { }^{k} Z(\{\varkappa\}) ; \quad{ }^{k} Z(\{\varkappa\})=\operatorname{Sp~e}^{k} \mathcal{H}
$$

and CGFs

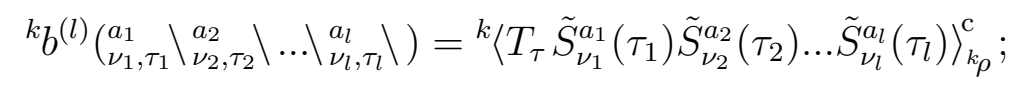

$$
\begin{aligned}
& \tilde{S}_{\nu}^{a}(\tau)=\mathrm{e}^{-\tau \cdot{ }^{k} \mathcal{H}} S_{\nu}^{a} \mathrm{e}^{\tau^{k}{ }^{k} \mathcal{H}}, \quad{ }^{k} \rho={ }^{k} \rho(\{\varkappa\})=\left[{ }^{k} Z(\{\varkappa\})\right]^{-1} \cdot \mathrm{e}^{k} \mathcal{H}
\end{aligned}
$$


of the reference system (2.3). In the present paper the ${ }^{k} \mathcal{F}$-function and pair CGFs will be calculated within the two-particle cluster approximation.

Assuming that the reference problem is solved in [26] the expansion of the free energy functional in the inverse radius of the long-range interactions was studied, for the systems described by the Hamiltonian (2.1) and expressions for temperature Green functions were obtained. Here we present only some of their results for nonuniform fields $\left(\Gamma_{\nu}^{a}=\Gamma^{a}\right)$ up to $r_{0}^{-d}$ in the long-range interactions.

The $\mathcal{F}$-function of the considered system reads

$$
\mathcal{F}(\{\Gamma\})={ }^{k} \mathcal{F}(\{\varkappa\})-\frac{N}{2} \sum_{a, b} J_{0}^{a b}\left\langle S^{a}\right\rangle_{\rho}\left\langle S^{b}\right\rangle_{\rho}-\frac{1}{2} \sum_{\omega_{n}, \vec{q}} \ln \operatorname{det}\left[\widehat{1}-{ }^{k} \widehat{b}^{(2)}\left(\vec{q}, \omega_{n}\right) \widehat{J}(\vec{q})\right],
$$

where

$$
J_{0}^{a b}=J^{a b}(\vec{q}=0), \quad \varkappa^{a}=\Gamma^{a}+\sum_{b} J_{0}^{a b}\left\langle S^{b}\right\rangle_{\rho} ;
$$

$\widehat{k}^{(2)}\left(\vec{q}, \omega_{n}\right)$ and $\widehat{J}(\vec{q})$ are matrices $3 \times 3$ in the indices $a, b$; their elements are Fourier transforms ${ }^{k} b^{(2)}\left(\begin{array}{c}a b \\ \vec{q}, \omega_{n}\end{array}\right)$ and $J^{a b}(\vec{q})$ of the pair CGFs of the reference system ${ }^{k} b^{(2)}\left(\begin{array}{l}a \\ \nu, \tau_{1}\end{array} \backslash_{\mu, \tau_{2}}^{b} \backslash\right)$ and of the long-range interactions $J_{\nu \mu}^{a b}$ (for uniform fields $\left(\varkappa_{\nu}^{a}=\varkappa^{a}\right)$ ).

For the pair CGFs $b^{(2)}\left(\underset{\nu, \tau_{1}}{a} \backslash{ }_{\mu, \tau_{2}}^{b} \backslash\right)$ in the frequency-momentum space, the following relation [26] holds

$$
\widehat{b}^{(2)}\left(\vec{q}, \omega_{n}\right)=\left[1-\widehat{M}\left(\vec{q}, \omega_{n}\right) \widehat{J}(\vec{q})\right]^{-1} \widehat{M}\left(\vec{q}, \omega_{n}\right) .
$$

Here we use the notation

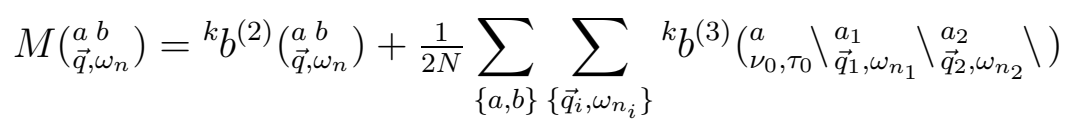

$$
\begin{aligned}
& \times^{k} b^{(3)}\left(\begin{array}{l}
b \\
\nu_{0}, \tau_{0}
\end{array} \backslash_{-\vec{q}_{1},-\omega_{n_{1}}}^{b_{1}} \backslash_{-\vec{q}_{2},-\omega_{n_{2}}}^{b_{2}} \backslash\right) \mathcal{R}\left(\begin{array}{l}
a_{1} b_{1} \\
{ }_{\vec{q}_{1},-\omega_{n_{1}}}
\end{array}\right) \mathcal{R}\left(\begin{array}{c}
a_{2} b_{2} \\
-\vec{q}_{2},-\omega_{n_{2}}
\end{array}\right) \\
& \times \delta\left(\vec{q}+\vec{q}_{1}+\vec{q}_{2}\right) \delta\left(\omega_{n}+\omega_{n_{1}}+\omega_{n_{2}}\right) \\
& +\frac{1}{2 N} \sum_{a_{1}, a_{2}} \sum_{\vec{q}_{1}, \omega_{n_{1}}}{ }^{k} b^{(4)}\left(\begin{array}{l}
a \\
\nu_{0}, \tau_{0}
\end{array} \backslash_{-\vec{q},-\omega_{n}}^{b} \backslash_{\vec{q}_{1}, \omega_{n_{1}}}^{a_{1}} \backslash_{-\vec{q}_{1},-\omega_{n_{1}}}^{a_{2}} \backslash\right) \mathcal{R}\left(\begin{array}{l}
a_{1} a_{2} \\
-\vec{q}_{1},-\omega_{n_{1}}
\end{array}\right),
\end{aligned}
$$

where

$$
\widehat{\mathcal{R}}\left(\vec{q}, \omega_{n}\right)=\widehat{J}(\vec{q})\left[1-{ }^{k} \widehat{b}^{(2)}\left(\vec{q}, \omega_{n}\right) \widehat{J}(\vec{q})\right]^{-1} \quad\left(\nu_{0}=0, \tau_{0}=0, \omega_{n}=2 \pi n \beta^{-1}\right)
$$

is the Fourier transform of the effective interaction in the considered system, whereas

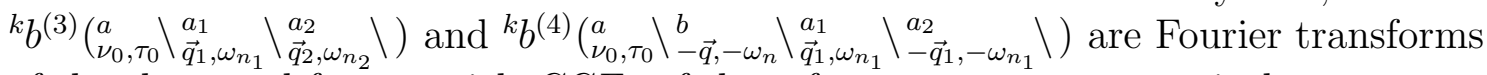
of the three and four-particle CGFs of the reference system, respectively.

We also present here an expression for the order parameter [26]

$$
\left\langle S^{a}\right\rangle=\left\langle S^{a}\right\rangle_{\rho}={ }^{k}\left\langle S^{a}\right\rangle_{k_{\rho}}+\frac{1}{2 N} \sum_{a_{1}, a_{2}} \sum_{\vec{q}, \omega_{n}}{ }^{k} b^{(3)}\left(\begin{array}{l}
a \\
\nu_{0}, \tau_{0}
\end{array} \backslash_{\vec{q}, \omega_{n}}^{a_{1}} \backslash \backslash_{-\vec{q},-\omega_{n}}^{a_{2}} \backslash\right) \mathcal{R}\left(\begin{array}{c}
a_{1} a_{2} \\
-\vec{q},-\omega_{n}
\end{array}\right) .
$$

Hence, we have general expressions for the free energy $\left(F=-k_{\mathrm{B}} T \mathcal{F}\right)$ and pair CGFs, and the equation for the order parameter in the $r_{0}^{-d}$ approximation. These expressions contain the free energy and correlation functions of the reference system. 


\section{Two-particle cluster approximation for short-range interactions}

\subsection{Problem formulation}

Our task in this section is to obtain the ${ }^{k} \mathcal{F}(\{\varkappa\})$-function, parameters ${ }^{k}\left\langle S_{\nu}^{a}\right\rangle_{k_{\rho}}$ and pair cumulant Green functions ${ }^{k} b^{(2)}\left(\begin{array}{l}a_{1} \backslash \tau_{1} \backslash \\ a_{2} \\ a_{2}\end{array} \backslash\right)$ of the reference pseudospin system, described by Hamiltonian ${ }^{k} H(\{\varkappa\})(2.3)$, in the two-particle cluster approximation in the short-range interactions.

Let us define the functional of the partition function logarithm (the ${ }^{k} \mathcal{F}(\{\varepsilon\})$ functional) of the reference model as [26]

$$
\begin{aligned}
& { }^{k} \mathcal{F}(\{\varepsilon\},\{\varkappa\})=\ln ^{k} Z(\{\varepsilon\},\{\varkappa\}) ; \\
& { }^{k} Z(\{\varepsilon\},\{\varkappa\})=\operatorname{Sp}\left[\mathrm{e}^{\mathrm{H}(\{\varepsilon\})} T_{\tau} \exp \left(\int_{0}^{1} \mathrm{~d} \tau^{k} \mathcal{H}(\tau,\{\varkappa\})\right)\right],
\end{aligned}
$$

where

$$
\begin{aligned}
& { }^{k} \mathcal{H}(\tau,\{\varkappa\})=\sum_{a} \sum_{\nu=1}^{N} \varkappa_{\nu, \tau}^{a} S_{\nu, \tau}^{a}+\frac{1}{2} \sum_{a, b} \sum_{\nu, \delta} K^{a b} S_{\nu, \tau}^{a} S_{\nu+\delta, \tau}^{b} ; \\
& \mathrm{H}(\{\varepsilon\})=\sum_{\nu=1}^{N} \mathrm{H}_{\nu}\left(\left\{\varepsilon_{\nu}\right\}\right) ; \quad \mathrm{H}_{\nu}\left(\left\{\varepsilon_{\nu}\right\}\right)=\sum_{a} \varepsilon_{\nu}^{a} S_{\nu}^{a} ; \quad A_{\tau}=\mathrm{e}^{-\tau \mathrm{H}(\{\varepsilon\})} A \mathrm{e}^{\tau \mathrm{H}(\{\varepsilon\})} .
\end{aligned}
$$

Dependence $\varkappa_{\nu, \tau}^{a}$ on $\tau$ here is necessary to perform functional differentiation with respect to $\varkappa_{\nu, \tau}^{a}[26]$. It should be noted, that since spin operators at different sites commute, and $\varkappa_{\nu}^{a}$ is a scalar, the quantity $A_{\tau}$ (if $A=S_{\nu}^{a}$ or $\varkappa_{\nu}^{a}$ ) can be written as

$$
A_{\tau}=\mathrm{e}^{-\tau \mathrm{H}_{\nu}\left(\left\{\varepsilon_{\nu}\right\}\right)} A \mathrm{e}^{\tau \mathrm{H}_{\nu}\left(\left\{\varepsilon_{\nu}\right\}\right)} .
$$

Starting from (3.1), we introduce functionals of CGFs of the reference system

$$
\begin{aligned}
& { }^{k} b^{(l)}\left(\underset{\nu_{1}, \tau_{1}}{a_{1}} \backslash_{\nu_{2}, \tau_{2}}^{a_{2}} \backslash \ldots \backslash{ }_{\nu_{l}, \tau_{l}}^{a_{l}} \backslash\{\varepsilon\}\right)={ }^{k}\left\langle T_{\tau} S_{\nu_{1}, \tau_{1}}^{a_{1}} S_{\nu_{2}, \tau_{2}}^{a_{2}} \ldots S_{\nu_{l}, \tau_{l}}^{a_{l}}\right\rangle_{k_{\rho}(\{\varepsilon\})}^{\mathrm{c}} ; \\
& { }^{k} \rho(\{\varepsilon\})=\frac{1}{{ }^{k} Z(\{\varepsilon\},\{\tilde{\varkappa}\})} \mathrm{e}^{\mathrm{H}(\{\varepsilon\})} \exp \left[\int_{0}^{1} \mathrm{~d} \tau^{k} \mathcal{H}(\tau,\{\varkappa\})\right] .
\end{aligned}
$$

They will be found using

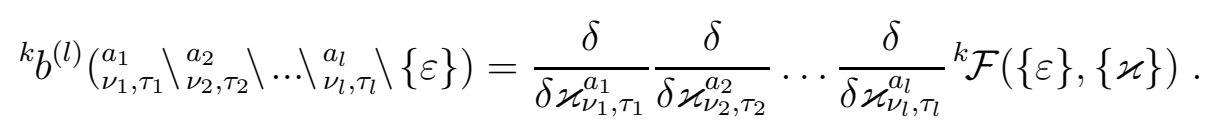

According to [26], the following relations between the ${ }^{k} \mathcal{F}(\{\varkappa\})$-function (2.9) and temperature CGFs (2.10) and their functionals hold

$$
\begin{aligned}
& { }^{k} \mathcal{F}(\{\varkappa\})={ }^{k} \mathcal{F}(\{\varepsilon\},\{\varkappa\})_{\substack{\varkappa_{\nu, \tau}^{a}=\varkappa_{\nu}^{a} \\
\varepsilon_{\nu}^{a}=0}} ;
\end{aligned}
$$

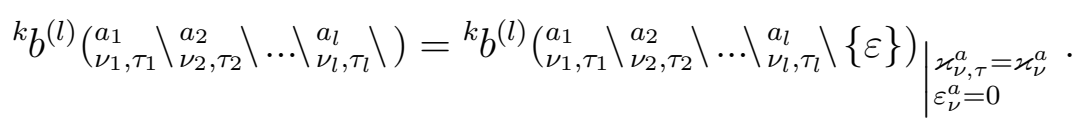


That is, calculation of the ${ }^{k} \mathcal{F}(\{\varkappa\})$-function and temperature CGFs is reduced to calculation of the ${ }^{k} \mathcal{F}(\{\varepsilon\},\{\varkappa\})$-functional.

\subsection{Cluster approximation. Free energy}

Let us calculate now the ${ }^{k} \mathcal{F}(\{\varepsilon\},\{\varkappa\})$-functional in the two-particle cluster approximation. We perform a cluster expansion, with the lattice being divided into the two-particle clusters $[47,54,55]$. As $\sum_{a}{ }^{r} \varphi_{\nu, \tau}^{a} S_{\nu, \tau}^{a}$ we denote an operator of the effective field created by the site $r$ and acting on the site $\nu$, provided that the site $r$ is the nearest neighbour of the site $\nu\left(r \in \pi_{\nu}\right)$. Obviously, the number of fields acting on an arbitrary site $\nu$

$$
\sum_{r \in \pi_{\nu}} \sum_{a}^{r} \varphi_{\nu, \tau}^{a} S_{\nu, \tau}^{a}
$$

is $z$ ( $z$ is the nearest neighbours number). After an identity transformation, the reference Hamiltonian (3.2) takes the form

$$
{ }^{k} \mathcal{H}(\tau,\{\varkappa, \varphi\})=\sum_{\nu} \mathcal{H}_{\nu}\left(\tau,\left\{\tilde{\varkappa}_{\nu}\right\}\right)+\sum_{(\nu, r)} \mathcal{U}_{\nu r}\left(\tau,\left\{{ }^{r} \varphi_{\nu},{ }^{\nu} \varphi_{r}\right\}\right)
$$

where

$$
\begin{aligned}
& \mathcal{H}_{\nu}\left(\tau,\left\{\tilde{\varkappa}_{\nu}\right\}\right)=\sum_{a} \tilde{\varkappa}_{\nu, \tau}^{a} S_{\nu, \tau}^{a} ; \quad \tilde{\varkappa}_{\nu, \tau}^{a}=\varkappa_{\nu, \tau}^{a}+\sum_{r \in \pi_{\nu}} \varphi_{\nu, \tau}^{a} \\
& \mathcal{U}_{\nu r}\left(\tau,\left\{{ }^{r} \varphi_{\nu},{ }^{\nu} \varphi_{r}\right\}\right)=\sum_{a}\left(-{ }^{r} \varphi_{\nu, \tau}^{a} S_{\nu, \tau}^{a}-{ }^{\nu} \varphi_{r, \tau}^{a} S_{r, \tau}^{a}+\sum_{b} K^{a b} S_{\nu, \tau}^{a} S_{r, \tau}^{b}\right)
\end{aligned}
$$

$\mathcal{H}_{\nu}\left(\tau,\left\{\tilde{\varkappa}_{\nu}\right\}\right)$ means $\mathcal{H}_{\nu}=\mathcal{H}_{\nu}\left(\tilde{\varkappa}_{\nu, \tau}^{a_{1}}, \tilde{\varkappa}_{\nu, \tau}^{a_{2}}, \tilde{\varkappa}_{\nu, \tau}^{a_{3}}\right)$. Hereafter, the arguments $\left\{\tilde{\varkappa}_{\nu}\right\},\left\{{ }^{r} \varphi_{\nu}\right\}$ will be frequently omitted.

Let us present the ${ }^{k} \mathcal{F}(\{\varepsilon\})$-functional (3.1) as

$$
\begin{aligned}
{ }^{k} \mathcal{F} & (\{\varepsilon\},\{\varkappa, \varphi\})= \\
& =\ln \operatorname{Sp}\left\{\mathrm{e}^{\mathrm{H}(\{\varepsilon\})} T_{\tau} \exp \left[\sum_{\nu=1}^{N} \int_{0}^{1} \mathrm{~d} \tau \mathcal{H}_{\nu}(\tau)\right] \exp \left[\sum_{(\nu, r)} \int_{0}^{1} \mathrm{~d} \tau \mathcal{U}_{\nu r}(\tau)\right]\right\} \\
& =\sum_{\nu} \mathcal{F}_{\nu}\left(\left\{\varepsilon_{\nu}\right\},\left\{\tilde{\mathcal{X}}_{\nu}\right\}\right)+\ln \left\langle T_{\tau} \exp \left(\sum_{(\nu, r)} \int_{0}^{1} \mathrm{~d} \tau \mathcal{U}_{\nu r}(\tau)\right)\right\rangle_{\rho_{0}(\{\varepsilon\})} .
\end{aligned}
$$

Here $\mathcal{F}_{\nu}\left(\left\{\varepsilon_{\nu}\right\}\right)$ is the so-called single-particle intracluster $\mathcal{F}(\{\varepsilon\})$-functional

$$
\begin{aligned}
& \mathcal{F}_{\nu}\left(\left\{\varepsilon_{\nu}\right\},\left\{\tilde{\varkappa}_{\nu}\right\}\right)=\ln Z_{\nu}\left(\left\{\varepsilon_{\nu}\right\},\left\{\tilde{\varkappa}_{\nu}\right\}\right) ; \\
& Z_{\nu}\left(\left\{\varepsilon_{\nu}\right\},\left\{\tilde{\varkappa}_{\nu}\right\}\right)=\operatorname{Sp}_{\boldsymbol{S}_{\nu}}\left\{\mathrm{e}^{\mathrm{H}_{\nu}\left(\left\{\varepsilon_{\nu}\right\}\right)} T_{\tau} \exp \left[\int_{0}^{1} \mathrm{~d} \tau \mathcal{H}_{\nu}\left(\tau,\left\{\tilde{\varkappa}_{\nu}\right\}\right)\right]\right\}
\end{aligned}
$$

and averaging is performed with the functional of the density matrix

$$
\begin{aligned}
& \rho_{0}(\{\varepsilon\})=\prod_{\nu} \rho_{\nu}\left(\left\{\varepsilon_{\nu}\right\}\right) ; \\
& \rho_{\nu}\left(\left\{\varepsilon_{\nu}\right\}\right)=\frac{1}{Z_{\nu}\left(\left\{\varepsilon_{\nu}\right\},\left\{\tilde{\varkappa}_{\nu}\right\}\right)} \mathrm{e}^{\mathrm{H}_{\nu}\left(\left\{\varepsilon_{\nu}\right\}\right)} \exp \left[\int_{0}^{1} \mathrm{~d} \tau \mathcal{H}_{\nu}\left(\tau,\left\{\tilde{\varkappa}_{\nu}\right\}\right)\right] .
\end{aligned}
$$


We restrict our consideration by the first order of the cluster expansion $[47,54$, 55]; this corresponds to the two-particle cluster approximation. Then the ${ }^{k \mathcal{F}}(\{\varepsilon\})$ functional becomes a sum of the single- and two-particle intracluster $\mathcal{F}(\{\varepsilon\})$-functionals

$$
\begin{aligned}
{ }^{k} \mathcal{F}(\{\varepsilon\},\{\varkappa, \varphi\}) & =\sum_{\nu} \mathcal{F}_{\nu}\left(\left\{\varepsilon_{\nu}\right\},\left\{\tilde{\varkappa}_{\nu}\right\}\right)+\sum_{(\nu, r)} \ln \left\langle T_{\tau} \exp \left(\int_{0}^{1} \mathrm{~d} \tau \mathcal{U}_{\nu r}(\tau)\right)\right\rangle_{\rho_{0}(\{\varepsilon\})} \\
& =(1-z) \sum_{\nu} \mathcal{F}_{\nu}\left(\left\{\varepsilon_{\nu}\right\},\left\{\tilde{\varkappa}_{\nu}\right\}\right)+\frac{1}{2} \sum_{\nu, r} \mathcal{F}_{\nu r}\left(\left\{\varepsilon_{\nu}, \varepsilon_{r}\right\},\left\{{ }^{r} \tilde{\varkappa}_{\nu},{ }^{\nu} \tilde{\varkappa}_{r}\right\}\right) .
\end{aligned}
$$

The two-particle $\mathcal{F}_{\nu r}\left(\left\{\varepsilon_{\nu}, \varepsilon_{r}\right\}\right)$-functional reads

$$
\begin{aligned}
& \mathcal{F}_{\nu r}\left(\left\{\varepsilon_{\nu}, \varepsilon_{r}\right\},\left\{{ }^{r} \tilde{\varkappa}_{\nu},{ }^{\nu} \tilde{\varkappa}_{r}\right\}\right)=\ln Z_{\nu r}\left(\left\{\varepsilon_{\nu}, \varepsilon_{r}\right\},\left\{{ }^{r} \tilde{\varkappa}_{\nu},{ }^{\nu} \tilde{\varkappa}_{r}\right\}\right) ; \\
& Z_{\nu r}\left(\left\{\varepsilon_{\nu}, \varepsilon_{r}\right\}\right)=\operatorname{Sp}_{S_{1}, S_{2}}\left\{\mathrm{e}^{\mathrm{H}_{\nu}\left(\left\{\varepsilon_{\nu}\right\}\right)+\mathrm{H}_{r}\left(\left\{\varepsilon_{r}\right\}\right)} T_{\tau} \exp \left[\int_{0}^{1} \mathrm{~d} \tau \mathcal{H}_{\nu r}\left(\tau,\left\{{ }^{r} \tilde{\varkappa}_{\nu},{ }^{\nu} \tilde{\varkappa}_{r}\right\}\right)\right]\right\} \\
& \mathcal{H}_{\nu r}\left(\tau,\left\{{ }^{r} \tilde{\varkappa}_{\nu},{ }^{\nu} \tilde{\varkappa}_{r}\right\}\right)=\mathcal{H}_{\nu}\left(\tau,\left\{\tilde{\varkappa}_{\nu}\right\}\right)+\mathcal{H}_{r}\left(\tau,\left\{\tilde{\varkappa}_{r}\right\}\right)+\mathcal{U}_{\nu r}(\tau) \\
& =\sum_{a}\left[\tilde{\varkappa}_{\nu, \tau}^{a} S_{\nu, \tau}^{a}+{ }^{\nu} \tilde{\varkappa}_{r, \tau}^{a} S_{r, \tau}^{a}+\sum_{b} K^{a b} S_{\nu, \tau}^{a} S_{r, \tau}^{b}\right] ; \\
& r_{\tilde{\varkappa}_{\nu, \tau}^{a}}^{a}=\tilde{\varkappa}_{\nu, \tau}^{a}-{ }^{r} \varphi_{\nu, \tau}^{a}=\mathcal{\varkappa}_{\nu, \tau}^{a}+\sum_{\substack{r^{\prime} \in \pi_{\nu} \\
r^{\prime} \neq r}} r^{\prime} \varphi_{\nu, \tau}^{a} .
\end{aligned}
$$

Putting $\varepsilon_{\nu}^{a}=0\left(\varkappa_{\nu, \tau}^{a}=\varkappa_{\nu}^{a},{ }^{r} \varphi_{\nu, \tau}^{a}={ }^{r} \varphi_{\nu}^{a}\right.$, see also (3.6)), and going to the uniform fields case $\varkappa_{\nu}^{a}=\varkappa^{a}\left({ }^{r} \varphi_{\nu}^{a}=\varphi^{a}\right)$, from (3.16) we obtain the ${ }^{k} \mathcal{F}$-function of the reference system in the TPCA for the uniform fields

$$
\begin{aligned}
& { }^{k} \mathcal{F}(\{\varkappa, \varphi\})=(1-z) N \mathcal{F}_{1}(\{\tilde{\varkappa}\})+\frac{N z}{2} \mathcal{F}_{12}(\{\tilde{\tilde{\varkappa}}\}) ; \\
& \mathcal{F}_{1}(\{\tilde{\varkappa}\})=\ln Z_{1}(\{\tilde{\varkappa}\}) ; \quad Z_{1}(\{\tilde{\varkappa}\})=\operatorname{Sp}_{S_{1}} \mathrm{e}^{\mathcal{H}_{1}(\{\tilde{\varkappa}\})} ; \\
& \mathcal{H}_{1}(\{\tilde{\varkappa}\})=\sum_{a} \tilde{\varkappa}^{a} S_{1}^{a} ; \quad \tilde{\varkappa}^{a}=\varkappa^{a}+z \varphi^{a} ; \\
& \mathcal{F}_{12}(\{\tilde{\tilde{\varkappa}}\})=\ln Z_{12}(\{\tilde{\tilde{\varkappa}}\}) ; \quad Z_{12}(\{\tilde{\tilde{\varkappa}}\})=\operatorname{Sp}_{S_{1}, S_{2}} \mathrm{e}^{\mathcal{H}_{12}(\{\tilde{\tilde{\varkappa}}\}) ;} \\
& \mathcal{H}_{12}(\{\tilde{\tilde{\varkappa}}\})=\sum_{a}\left[\tilde{\tilde{\varkappa}}^{a}\left(S_{1}^{a}+S_{2}^{a}\right)+\sum_{b} K^{a b} S_{1}^{a} S_{2}^{b}\right] ; \quad \tilde{\tilde{\varkappa}}^{a}=\varkappa^{a}+(z-1) \varphi^{a} .
\end{aligned}
$$

\subsection{System of equations for single-particle distribution functions and vari- ational parameters}

Let us now find equations for functionals ${ }^{k}\left\langle T_{\tau} S_{\nu, \tau}^{a}\right\rangle_{k \rho(\{\varepsilon\})}$ and for cluster fields ${ }^{r} \varphi_{\nu, \tau}^{a}$. From (3.5) we obtain 


$$
{ }^{k}\left\langle T_{\tau} S_{\mu, \tau}^{a}\right\rangle_{k \rho(\{\varepsilon\})}=\frac{\partial^{k} \mathcal{F}(\{\varepsilon\})}{\partial \varkappa_{\mu, \tau}^{a}}+\sum_{\nu} \sum_{r \in \pi_{\nu}} \sum_{b} \int_{0}^{1} \mathrm{~d} \tau^{\prime} \frac{\partial^{k} \mathcal{F}(\{\varepsilon\})}{\partial^{r} \varphi_{\nu, \tau^{\prime}}^{b}} \cdot \frac{\delta^{r} \varphi_{\nu, \tau^{\prime}}^{b}}{\delta \varkappa_{\mu, \tau}^{a}}
$$

Starting from the fact that the ${ }^{k} \mathcal{F}(\{\varepsilon\})$-functional (3.16) is a sum of the single- and two-particle $\mathcal{F}(\{\varepsilon\})$-functionals, we get

$$
\begin{aligned}
& \frac{\partial^{k} \mathcal{F}(\{\varepsilon\})}{\partial \varkappa_{\nu, \tau}^{a}}=(1-z) \mathcal{F}_{\nu}^{(1)}\left({ }_{\tau}^{a} \mid\left\{\varepsilon_{\nu}\right\}\right)+\sum_{r \in \pi_{\nu}} \mathcal{F}_{\nu r}^{(1,0)}\left(\underset{\tau}{a} \mid\left\{\varepsilon_{\nu}, \varepsilon_{r}\right\}\right) ; \\
& \frac{\partial^{k} \mathcal{F}(\{\varepsilon\})}{\partial^{r_{1}} \varphi_{\nu, \tau}^{a}}=(1-z) \mathcal{F}_{\nu}^{(1)}\left({ }_{\tau}^{a} \mid\left\{\varepsilon_{\nu}\right\}\right)+\sum_{\substack{r \in \pi_{\nu} \\
r \neq r_{1}}} \mathcal{F}_{\nu r}^{(1,0)}\left({ }_{\tau}^{a} \mid\left\{\varepsilon_{\nu}, \varepsilon_{r}\right\}\right), \quad\left(r_{1} \in \pi_{\nu}\right) .
\end{aligned}
$$

Here we introduce the notations

$$
\begin{aligned}
& \mathcal{F}_{\nu}^{(k)}\left(\begin{array}{cccc}
a_{1} & a_{2} \\
\tau_{1} & \tau_{2}
\end{array}\left|\cdots c_{\tau_{k}}^{a_{k}}\right|\left\{\varepsilon_{\nu}\right\},\left\{\tilde{\varkappa}_{\nu}\right\}\right)=\frac{\partial}{\partial \tilde{\varkappa}_{\nu, \tau_{1}}^{a_{1}}} \cdot \frac{\partial}{\partial \tilde{\varkappa}_{\nu, \tau_{2}}^{a_{2}}} \cdots \frac{\partial}{\partial \tilde{\varkappa}_{\nu, \tau_{k}}^{a_{k}}} \mathcal{F}_{\nu}\left(\left\{\varepsilon_{\nu}\right\},\left\{\tilde{\varkappa}_{\nu}\right\}\right),
\end{aligned}
$$

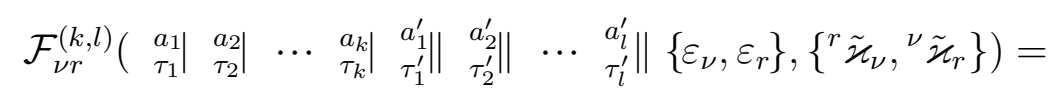

$$
\begin{aligned}
& =\frac{\partial}{\partial{ }^{r} \tilde{\varkappa}_{\nu, \tau_{1}}^{a_{1}}} \cdots \frac{\partial}{\partial{ }^{r} \tilde{\varkappa}_{\nu, \tau_{k}}^{a_{k}}} \cdot \frac{\partial}{\partial{ }^{\nu} \tilde{\mathcal{\varkappa}}_{r, \tau_{1}^{\prime}}^{a_{1}^{\prime}}} \cdots \frac{\partial}{\partial \nu \tilde{\varkappa}_{r, \tau_{l}^{\prime}}^{a_{l}^{\prime}}} \mathcal{F}_{\nu r}\left(\left\{\varepsilon_{\nu}, \varepsilon_{r}\right\},\left\{{ }^{r} \tilde{\mathcal{\varkappa}}_{\nu},{ }^{\nu} \tilde{\varkappa}_{r}\right\}\right) .
\end{aligned}
$$

From the explicit form of the intracluster $\mathcal{F}(\{\varepsilon\})$-functionals (3.12), (3.17) it follows that

$$
\begin{aligned}
& \mathcal{F}_{\nu}^{(k)}\left(\begin{array}{cccc}
a_{1} & a_{\tau_{1}} \\
\tau_{2}
\end{array}\left|\cdots a_{\tau_{k}}^{a_{k}}\right|\left\{\varepsilon_{\nu}\right\},\left\{\tilde{\varkappa}_{\nu}\right\}\right)=\left\langle T_{\tau} S_{\nu \tau_{1}}^{a_{1}} S_{\nu \tau_{2}}^{a_{2}} \cdots S_{\nu \tau_{k}}^{a_{k}}\right\rangle_{\rho_{\nu}\left(\left\{\varepsilon_{\nu}\right\}\right)}^{\mathrm{c}} ;
\end{aligned}
$$

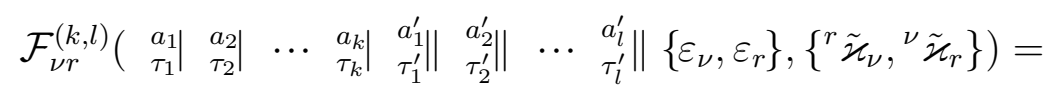

$$
\begin{aligned}
& =\left\langle T_{\tau} S_{\nu \tau_{1}}^{a_{1}} S_{\nu \tau_{2}}^{a_{2}} \cdots S_{\nu \tau_{k}}^{a_{k}} S_{r \tau_{1}^{\prime}}^{a_{1}^{\prime}} S_{r \tau_{2}^{\prime}}^{a_{2}^{\prime}} \cdots S_{r \tau_{l}}^{a_{l}^{\prime}}\right\rangle_{\rho_{\nu r}}^{\mathrm{c}}\left(\left\{\varepsilon_{\nu}, \varepsilon_{r}\right\}\right),
\end{aligned}
$$

where the averagings are performed with the density matrix functionals (3.15) and

$$
\rho_{\nu r}\left(\left\{\varepsilon_{\nu}, \varepsilon_{r}\right\}\right)=\frac{\mathrm{e}^{\mathrm{H}_{\nu}\left(\left\{\varepsilon_{\nu}\right\}\right)+\mathrm{H}_{r}\left(\left\{\varepsilon_{r}\right\}\right)}}{Z_{\nu r}\left(\left\{\varepsilon_{\nu}, \varepsilon_{r}\right\},\left\{{ }^{r} \tilde{\varkappa}_{\nu},{ }^{\nu} \tilde{\varkappa}_{r}\right\}\right)} \exp \left[\int_{0}^{1} \mathrm{~d} \tau \mathcal{H}_{\nu r}\left(\tau,\left\{{ }^{r} \tilde{\varkappa}_{\nu},{ }^{\nu} \tilde{\varkappa}_{r}\right\}\right)\right] .
$$

Hereafter, the functionals (3.31) and (3.32) will be called the single-particle and two-particle intracluster functionals of CGF, respectively.

Similar to (3.6), from (3.12) and (3.17) one can obtain expressions relating the intracluster $\mathcal{F}$-functions with their functionals

$$
\begin{aligned}
& \mathcal{F}_{\nu}\left(\left\{\tilde{\varkappa}_{\nu}\right\}\right)=\left.\mathcal{F}_{\nu}\left(\left\{\varepsilon_{\nu}\right\},\left\{\tilde{\varkappa}_{\nu}\right\}\right)\right|_{\substack{\tilde{\varkappa}_{\nu, \tau}^{a}=\tilde{\varkappa}_{\nu}^{a} \\
\varepsilon_{\nu}^{a}=0}} ; \\
& \mathcal{F}_{\nu r}\left(\left\{{ }^{r} \tilde{\varkappa}_{\nu},{ }^{\nu} \tilde{\varkappa}_{r}\right\}\right)=\mathcal{F}_{\nu r}\left(\left\{\varepsilon_{\nu}, \varepsilon_{r}\right\},\left.\left\{{ }^{r} \tilde{\varkappa}_{\nu},{ }^{\nu} \tilde{\varkappa}_{r}\right\}\right|_{\mid \begin{array}{l}
r_{\tilde{\varkappa}_{\nu, \tau}^{a}}^{a}=\tilde{\varkappa}_{\nu}^{a}, \nu \tilde{\varkappa}_{r, \tau}^{a}=\tilde{\varkappa}_{r}^{a} \\
\varepsilon_{\nu}^{a}=\varepsilon_{r}^{a}=0
\end{array}} .\right.
\end{aligned}
$$


One can also derive expressions relating the single-particle and two-particle intracluster CGFs with their functionals

$$
\begin{aligned}
& \left\langle T_{\tau} \bar{S}_{\nu}^{a_{1}}\left(\tau_{1}\right) \bar{S}_{\nu}^{a_{2}}\left(\tau_{2}\right) \ldots \bar{S}_{\nu}^{a_{k}}\left(\tau_{k}\right)\right\rangle_{\rho_{\nu}}^{\mathrm{c}}=\left.\left\langle T_{\tau} S_{\nu, \tau_{1}}^{a_{1}} S_{\nu, \tau_{2}}^{a_{2}} \ldots S_{\nu, \tau_{k}}^{a_{k}}\right\rangle_{\rho_{\nu}\left(\left\{\varepsilon_{\nu}\right\}\right)}^{\mathrm{c}}\right|_{\substack{\tilde{\varkappa}_{\nu, \tau}^{a}=\tilde{\varkappa}_{\nu}^{a} \\
\varepsilon_{\nu}^{a}=0}} \\
& \left\langle T_{\tau} \overline{\bar{S}}_{\nu}^{a_{1}}\left(\tau_{1}\right) \overline{\bar{S}}_{\nu}^{a_{2}}\left(\tau_{2}\right) \cdots \overline{\bar{S}}_{\nu}^{a_{k}}\left(\tau_{k}\right) \overline{\bar{S}}_{r}^{a_{1}^{\prime}}\left(\tau_{1}^{\prime}\right) \overline{\bar{S}}_{r}^{a_{2}^{\prime}}\left(\tau_{2}^{\prime}\right) \cdots \overline{\bar{S}}_{r}^{a_{l}^{\prime}}\left(\tau_{l}^{\prime}\right)\right\rangle_{\rho_{\nu r}}^{\mathrm{c}}=
\end{aligned}
$$

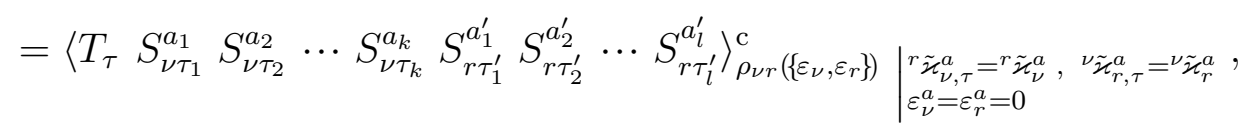

where

$$
\begin{aligned}
& \bar{S}_{\nu}^{a}(\tau)=\mathrm{e}^{-\tau \mathcal{H}_{\nu}} S_{\nu}^{a} \mathrm{e}^{\tau \mathcal{H}_{\nu}} ; \quad \rho_{\nu}=\frac{\mathrm{e}^{\mathcal{H}_{\nu}}}{\operatorname{Sp}\left(\mathrm{e}^{\mathcal{H}_{\nu}}\right)} ; \quad \mathcal{H}_{\nu}\left(\left\{\tilde{\varkappa}_{\nu}\right\}\right)=\sum_{a} \tilde{\varkappa}_{\nu}^{a} S_{\nu}^{a} ; \\
& \overline{\bar{S}}_{\nu}^{a}(\tau)=\mathrm{e}^{-\tau \mathcal{H}_{\nu r}} S_{\nu}^{a} \mathrm{e}^{\tau \mathcal{H}_{\nu r}} ; \quad \rho_{\nu r}=\frac{\mathrm{e}^{\mathcal{H}_{\nu r}}}{\mathrm{Sp}\left(\mathrm{e}^{\mathcal{H}_{\nu r}}\right)} ; \\
& \mathcal{H}_{\nu r}\left(\left\{{ }^{r} \tilde{\varkappa}_{\nu},{ }^{\nu} \tilde{\varkappa}_{r}\right\}\right)=\sum_{a}\left[{ }^{r} \tilde{\varkappa}_{\nu}^{a} S_{\nu}^{a}+{ }^{\nu} \tilde{\varkappa}_{r}^{a} S_{r}^{a}+\sum_{b} K^{a b} S_{\nu}^{a} S_{r}^{b}\right] .
\end{aligned}
$$

From equations (3.26)-(3.28), taking into account the condition of the extremum of the ${ }^{k} \mathcal{F}(\{\varepsilon\})$-functional with respect to ${ }^{r} \varphi_{\nu, \tau}^{a}$

$$
\frac{\partial^{k} \mathcal{F}(\{\varepsilon\},\{\varkappa, \varphi\})}{\partial^{r} \varphi_{\nu, \tau}^{a}}=0,
$$

we obtain the system of equations for the functionals ${ }^{k}\left\langle T_{\tau} S_{\nu, \tau}^{a}\right\rangle_{k_{\rho}(\{\varepsilon\})}$ and cluster fields ${ }^{r} \varphi_{\nu, \tau}^{a}$

$$
\begin{aligned}
& { }^{k}\left\langle T_{\tau} S_{\nu, \tau}^{a}\right\rangle_{k_{\rho}(\{\varepsilon\})}=\mathcal{F}_{\nu}^{(1)}\left({ }_{\tau}^{a} \mid\left\{\varepsilon_{\nu}\right\},\left\{\tilde{\varkappa}_{\nu}\right\}\right), \\
& \mathcal{F}_{\nu}^{(1)}\left({ }_{\tau}^{a} \mid\left\{\varepsilon_{\nu}\right\},\left\{\tilde{\varkappa}_{\nu}\right\}\right)=\mathcal{F}_{\nu r}^{(1,0)}\left(\begin{array}{c}
a \\
\tau
\end{array} \mid\left\{\varepsilon_{\nu}, \varepsilon_{r}\right\},\left\{{ }^{r} \tilde{\varkappa}_{\nu},{ }^{\nu} \tilde{\varkappa}_{r}\right\}\right) .
\end{aligned}
$$

One can see that equation (3.42) for ${ }^{r} \varphi_{\nu, \tau}^{a}$, obtained from the ${ }^{k} \mathcal{F}(\{\varepsilon\})$-functional extremum condition (3.40), is equivalent to equations

$$
\left\langle T_{\tau} S_{\nu, \tau}^{a}\right\rangle_{\rho_{\nu}\left(\left\{\varepsilon_{\nu}\right\}\right)}=\left\langle T_{\tau} S_{\nu, \tau}^{a}\right\rangle_{\rho_{\nu r}\left(\left\{\varepsilon_{\nu}, \varepsilon_{r}\right\}\right)} .
$$

That is, in the present approximation (see (3.36)-(3.39)) the relations between the density matrices are not violated:

$$
\left\langle S_{\nu}^{a}\right\rangle_{\rho_{\nu}}=\left\langle S_{\nu}^{a}\right\rangle_{\rho_{\nu r}} \quad \Longrightarrow \quad \rho_{\nu}=\operatorname{Sp}_{\boldsymbol{S}_{r}} \rho_{\nu r}
$$

Putting $\varepsilon_{\nu}^{a}=0\left(\varkappa_{\nu, \tau}^{a}=\varkappa_{\nu}^{a},{ }^{r} \varphi_{\nu, \tau}^{a}={ }^{r} \varphi_{\nu}^{a}\right.$, see also (3.7), (3.36), (3.37)), taking into account the following relations

$$
\left\langle S_{\nu}^{a}\right\rangle_{\rho_{\nu}}=\frac{\partial \mathcal{F}_{\nu}\left(\left\{\tilde{\varkappa}_{\nu}\right\}\right)}{\partial \tilde{\varkappa}_{\nu}^{a}}, \quad\left\langle S_{\nu}^{a}\right\rangle_{\rho_{\nu r}}=\frac{\partial \mathcal{F}_{\nu r}\left(\left\{{ }^{r} \tilde{\varkappa}_{\nu},{ }^{\nu} \tilde{\varkappa}_{r}\right\}\right)}{\partial{ }^{r} \tilde{\varkappa}_{\nu}^{a}}
$$


and going to the uniform fields case $\varkappa_{\nu}^{a}=\varkappa^{a}\left({ }^{r} \varphi_{\nu}^{a}=\varphi^{a}, \tilde{\varkappa}_{\nu}^{a}=\tilde{\varkappa}^{a},{ }^{r} \tilde{\varkappa}_{\nu}^{a}=\tilde{\tilde{\varkappa}}^{a}\right)$, from (3.41), (3.42) we obtain the system of equations for the single-particle distribution functions ${ }^{k}\left\langle S^{a}\right\rangle_{k_{\rho}}$ and cluster fields $\varphi^{a}$ in the TPCA for the uniform fields case.

$$
\begin{aligned}
& { }^{k}\left\langle S^{a}\right\rangle_{k_{\rho}}=\frac{\partial \mathcal{F}_{1}(\{\tilde{\varkappa}\})}{\partial \tilde{\varkappa}^{a}}, \\
& \frac{\partial \mathcal{F}_{1}(\{\tilde{\varkappa}\})}{\partial \tilde{\varkappa}^{a}}=\frac{1}{2} \frac{\partial \mathcal{F}_{12}(\{\tilde{\tilde{\varkappa}}\})}{\partial \tilde{\tilde{\varkappa}}^{a}} .
\end{aligned}
$$

Here $\mathcal{F}_{1}(\{\tilde{\varkappa}\})$ and $\mathcal{F}_{12}(\{\tilde{\tilde{\varkappa}}\})$ are the single-particle and two-particle intracluster $\mathcal{F}$ functions for the uniform fields case (3.22), (3.24). The factor $\frac{1}{2}$ in the right hand side of equation (3.44) arose at going from the partial derivative of the $\mathcal{F}_{\nu r}\left(\left\{{ }^{r} \tilde{\varkappa}_{\nu},{ }^{\nu} \tilde{\varkappa}_{r}\right\}\right)$ function with respect to ${ }^{r} \tilde{\varkappa}_{\nu}^{a}$ in the non-uniform fields case to the partial derivative of the $\mathcal{F}_{12}(\{\tilde{\tilde{\varkappa}}\})$-function with respect to $\tilde{\tilde{\varkappa}}^{a}$ in the uniform fields case.

\subsection{Pair distribution functions}

Let us briefly discuss the method of calculation of the pair CGFs functionals of the reference system presented in [54], based on the technique developed in [47] for the Ising model. Starting from (3.5) and (3.41), we obtain an expression for the pair CGF functional

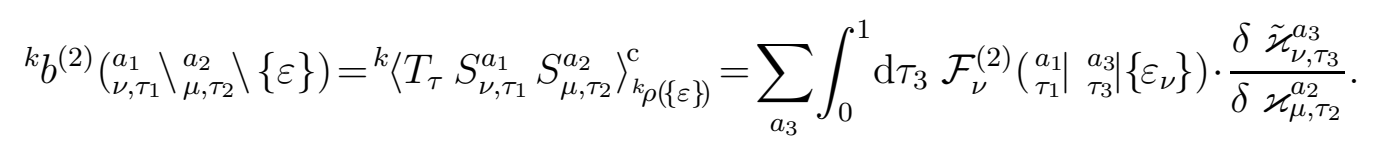

Having in mind the calculations of the pair CGFs (see (3.7)) for the uniform fields case, and since for specific systems single-particle intracluster pair CGFs $\left\langle T_{\tau} \bar{S}_{\nu}^{a_{1}}\left(\tau_{1}\right) \bar{S}_{\nu}^{a_{2}}\left(\tau_{2}\right)\right\rangle_{\rho_{\nu}}^{\mathrm{c}}($ see $(3.31),(3.36))$ in uniform fields case can be calculated directly, we need to obtain an equation for $\delta \tilde{\varkappa}_{\nu, \tau_{3}}^{a_{3}} / \delta \varkappa_{\mu, \tau_{2}}^{a_{2}}[47,54,56]$.

We introduce the notations

$$
\tilde{\varkappa}_{\nu \mu}^{\prime}\left(\left.\begin{array}{c}
a_{3} \\
\tau_{3}
\end{array}\right|_{\tau_{2}} ^{a_{2}}\right)=\frac{\delta \tilde{\varkappa}_{\nu, \tau_{3}}^{a_{3}}}{\delta \varkappa_{\mu, \tau_{2}}^{a_{2}}} ; \quad{ }^{r} \tilde{\varkappa}_{\nu \mu}^{\prime}\left(\left.\begin{array}{c}
a_{3} \\
\tau_{3}
\end{array}\right|_{\tau_{2}} ^{a_{2}}\right)=\frac{\delta{ }^{r} \tilde{\varkappa}_{\nu, \tau_{3}}^{a_{3}}}{\delta \varkappa_{\mu, \tau_{2}}^{a_{2}}} ; \quad r^{r} \varphi_{\nu \mu}^{\prime}\left(\left.\left.\right|_{\tau_{3}} ^{a_{3}}\right|_{\tau_{2}} ^{a_{2}}\right)=\frac{\delta^{r} \varphi_{\nu, \tau_{3}}^{a_{3}}}{\delta \varkappa_{\mu, \tau_{2}}^{a_{2}}} .
$$

Taking the functional derivative $\delta / \delta \varkappa_{\mu, \tau_{2}}^{a_{2}}$ from both sides of equation (3.42), and taking into account the relation

$$
r_{\tilde{\varkappa}_{\nu \mu}}^{\prime}\left(\left.a_{\tau_{3}}^{a_{3}}\right|_{\tau_{2}} ^{a_{2}}\right)=\tilde{\varkappa}_{\nu \mu}^{\prime}\left(\left.a_{\tau_{3}}^{a_{3}}\right|_{\tau_{2}} ^{a_{2}}\right)-{ }^{r} \varphi_{\nu \mu}^{\prime}\left(\left.\begin{array}{l}
a_{3} \\
\tau_{3}
\end{array}\right|_{\tau_{2}} ^{a_{2}}\right)
$$

(see (3.20)), we obtain

$$
\begin{aligned}
& \sum_{a_{3}} \int_{0}^{1} \mathrm{~d} \tau_{3} \mathcal{F}_{\nu}^{(2)}\left(\begin{array}{cc}
a_{1} & a_{3} \\
\tau_{1} & \tau_{3}
\end{array} \mid\left\{\varepsilon_{\nu}\right\}\right) \cdot \tilde{\varkappa}_{\nu \mu}^{\prime}\left(\begin{array}{c}
a_{3} \\
\tau_{3}
\end{array} \mid \begin{array}{c}
a_{2} \\
\tau_{2}
\end{array}\right)= \\
& =\sum_{a_{3}} \int_{0}^{1} \mathrm{~d} \tau_{3} \mathcal{F}_{\nu r}^{(2,0)}\left(\begin{array}{cc}
a_{1} & a_{3} \\
\tau_{1} & a_{3}
\end{array} \mid\left\{\varepsilon_{\nu}, \varepsilon_{r}\right\}\right)\left[\tilde{\varkappa}_{\nu \mu}^{\prime}\left(\begin{array}{l}
a_{3} \\
\tau_{3}
\end{array} \mid a_{\tau_{2}}^{a_{2}}\right)-{ }^{r} \varphi_{\nu \mu}^{\prime}\left(\left.\begin{array}{l}
a_{3} \\
\tau_{3}
\end{array}\right|_{\tau_{2}} ^{a_{2}}\right)\right] \\
& +\sum_{a_{3}} \int_{0}^{1} \mathrm{~d} \tau_{3} \mathcal{F}_{\nu r}^{(1,1)}\left(\begin{array}{cc}
a_{1} & a_{3} \\
\tau_{1} & a_{3}
\end{array} \mid\left\{\varepsilon_{\nu}, \varepsilon_{r}\right\}\right)\left[\tilde{\varkappa}_{r \mu}^{\prime}\left(\left.\begin{array}{l}
\left(a_{3}\right. \\
\tau_{3}
\end{array}\right|_{\tau_{2}} ^{a_{2}}\right)-{ }^{\nu} \varphi_{r \mu}^{\prime}\left(\begin{array}{l}
a_{3} \\
\tau_{3}
\end{array} \mid \begin{array}{l}
a_{2} \\
\tau_{2}
\end{array}\right)\right] .
\end{aligned}
$$


Going to a matrix form in (3.48) ${ }^{1}$ and performing some transformations, we obtain:

$$
\begin{aligned}
& {\left[\widehat{\mathcal{F}}_{\nu r}^{(2,0)}\left(\left\{\varepsilon_{\nu}, \varepsilon_{r}\right\}\right)-\widehat{\mathcal{F}}_{\nu}^{(2)}\left(\left\{\varepsilon_{\nu}\right\}\right)\right] \cdot \widehat{\hat{\varkappa}}_{\nu \mu}^{\prime}+\widehat{\mathcal{F}}_{\nu r}^{(1,1)}\left(\left\{\varepsilon_{\nu}, \varepsilon_{r}\right\}\right) \cdot \widehat{\tilde{\varkappa}}_{r \mu}^{\prime}=} \\
& =\widehat{\mathcal{F}}_{\nu r}^{(2,0)}\left(\left\{\varepsilon_{\nu}, \varepsilon_{r}\right\}\right) \cdot{ }^{r} \widehat{\varphi}_{\nu \mu}^{\prime}+\widehat{\mathcal{F}}_{\nu r}^{(1,1)}\left(\left\{\varepsilon_{\nu}, \varepsilon_{r}\right\}\right) \cdot{ }^{\nu} \widehat{\varphi}_{r \mu}^{\prime} .
\end{aligned}
$$

Introducing the notations

$$
\begin{aligned}
& \widehat{f}_{\nu r}=\left[\widehat{\mathcal{F}}_{\nu r}^{(2,0)}\left(\left\{\varepsilon_{\nu}, \varepsilon_{r}\right\}\right)\right]^{-1} \cdot \widehat{\mathcal{F}}_{\nu r}^{(1,1)}\left(\left\{\varepsilon_{\nu}, \varepsilon_{r}\right\}\right) ; \\
& \widehat{v}_{\nu r}=\left[\widehat{\mathcal{F}}_{\nu r}^{(2,0)}\left(\left\{\varepsilon_{\nu}, \varepsilon_{r}\right\}\right)\right]^{-1} \cdot \widehat{\mathcal{F}}_{\nu}^{(2)}\left(\left\{\varepsilon_{\nu}, \varepsilon_{r}\right\}\right),
\end{aligned}
$$

we rewrite equation (3.49) as

$$
{ }^{r} \widehat{\varphi}_{\nu \mu}^{\prime}+\widehat{f}_{\nu r} \cdot{ }^{\nu} \widehat{\varphi}_{r \mu}^{\prime}=\widehat{f}_{\nu r} \cdot \widehat{\tilde{\varkappa}}_{r \mu}^{\prime}+\left(\widehat{1}-\widehat{v}_{\nu r}\right) \widehat{\tilde{\varkappa}}_{\nu \mu}^{\prime}, \quad\left(r \in \pi_{\nu}\right) .
$$

We obtain an equation (3.51) with unknown ${ }^{r} \varphi_{\nu \mu}^{\prime},{ }^{\nu} \varphi_{r \mu}^{\prime}$. One more linearly independent equation still should be derived. After changing indices $r \rightleftharpoons \nu$, we get

$$
\nu \widehat{\varphi}_{r \mu}^{\prime}+\widehat{f}_{r \nu} \cdot r \widehat{\varphi}_{\nu \mu}^{\prime}=\widehat{f}_{r \nu} \cdot \widehat{\tilde{\varkappa}}_{\nu \mu}^{\prime}+\left(\widehat{1}-\widehat{v}_{r \nu}\right) \widehat{\tilde{\varkappa}}_{r \mu}^{\prime}, \quad\left(\nu \in \pi_{r}\right) .
$$

One can easily see that (3.51) and (3.52) are a system of equations for ${ }^{r} \varphi_{\nu \mu}^{\prime},{ }^{\nu} \varphi_{r \mu}^{\prime}$. Summing up over $r \in \pi_{\nu}$ in (3.51) and taking into account the fact that

$$
\widehat{\tilde{\varkappa}}_{\nu \mu}^{\prime}=\delta_{\nu \mu} \cdot \widehat{1}+\sum_{r \in \pi_{\nu}}^{r} \widehat{\varphi}_{\nu \mu}^{\prime}
$$

from the system of equations $(3.51),(3.52)$ one obtains a closed equation for $\tilde{\varkappa}_{\nu \mu}^{\prime}$

$$
\begin{gathered}
\left\{\widehat{1}+\sum_{r \in \pi_{\nu}} \widehat{f}_{\nu r}\left[\widehat{1}-\widehat{f}_{r \nu} \cdot \widehat{f}_{\nu r}\right]^{-1}\left[\widehat{f}_{r \nu}-\widehat{f}_{\nu r}^{-1}\left(\widehat{1}-\widehat{v}_{\nu r}\right)\right]\right\} \widehat{\tilde{\varkappa}}_{\nu \mu}^{\prime}= \\
=\delta_{\nu \mu} \cdot \widehat{1}+\sum_{r \in \pi_{\nu}} \widehat{f}_{\nu r}\left[\widehat{1}-\widehat{f}_{r \nu} \cdot \widehat{f}_{\nu r}\right]^{-1} \widehat{v}_{r \nu} \cdot \widehat{\tilde{\varkappa}}_{r \mu}^{\prime}
\end{gathered}
$$

\footnotetext{
${ }^{1}$ Here the matrices have a block structure; for instance in terms of $(x, y, z)$ :
}

$$
\widehat{\mathcal{F}}_{\nu}^{(2)}\left(\left\{\varepsilon_{\nu}\right\}\right)=\left(\begin{array}{lll}
\mathcal{F}_{\nu}^{(2)}\left(x|x|\left\{\varepsilon_{\nu}\right\}\right) & \mathcal{F}_{\nu}^{(2)}\left(x|y|\left\{\varepsilon_{\nu}\right\}\right) & \mathcal{F}_{\nu}^{(2)}\left(x|z|\left\{\varepsilon_{\nu}\right\}\right) \\
\mathcal{F}_{\nu}^{(2)}\left(y|x|\left\{\varepsilon_{\nu}\right\}\right) & \mathcal{F}_{\nu}^{(2)}\left(y|y|\left\{\varepsilon_{\nu}\right\}\right) & \mathcal{F}_{\nu}^{(2)}\left(y|z|\left\{\varepsilon_{\nu}\right\}\right) \\
\mathcal{F}_{\nu}^{(2)}\left(z|x|\left\{\varepsilon_{\nu}\right\}\right) & \mathcal{F}_{\nu}^{(2)}\left(z|y|\left\{\varepsilon_{\nu}\right\}\right) & \mathcal{F}_{\nu}^{(2)}\left(z|z|\left\{\varepsilon_{\nu}\right\}\right)
\end{array}\right),
$$

where the submatrices

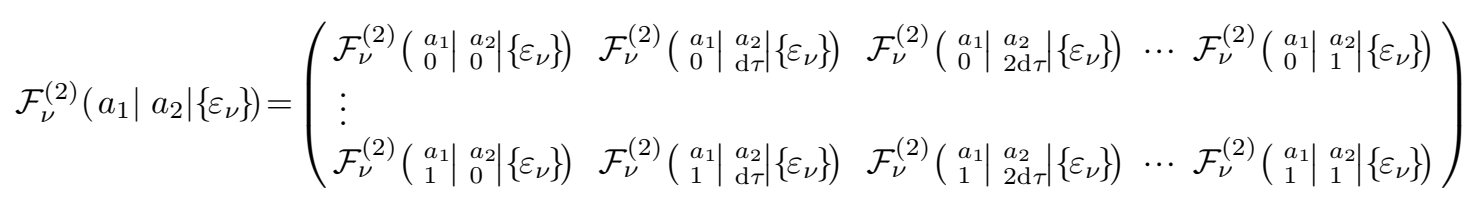

are the $\mathrm{M} \times \mathrm{M}$ matrices $\left(\mathrm{M}=\frac{1}{\mathrm{~d} \tau}+1\right)$. At $\mathrm{d} \tau \longrightarrow 0 \mathrm{M} \longrightarrow \infty$. 
Let us put $\varepsilon_{\nu}^{a}=0$ and go to the uniform fields case $\varkappa_{\nu}^{a}=\varkappa^{a}$

$$
\begin{array}{ll}
\widehat{\mathcal{F}}_{\nu r}^{(2,0)}\left(\left\{\varepsilon_{\nu}, \varepsilon_{r}\right\}\right)=\widehat{\mathcal{F}}_{(\nu-r)}^{(2,0)}\left(\left\{\varepsilon_{\nu}, \varepsilon_{r}\right\}\right) \longrightarrow \widehat{\mathcal{F}}_{12}^{(2,0)} ; & \widehat{\mathcal{F}}_{\nu}^{(2)}\left(\left\{\varepsilon_{\nu}\right\}\right) \longrightarrow \widehat{\mathcal{F}}_{1}^{(2)} ; \\
\widehat{\mathcal{F}}_{\nu r}^{(1,1)}\left(\left\{\varepsilon_{\nu}, \varepsilon_{r}\right\}\right)=\widehat{\mathcal{F}}_{(\nu-r)}^{(1,1)}\left(\left\{\varepsilon_{\nu}, \varepsilon_{r}\right\}\right) \longrightarrow \widehat{\mathcal{F}}_{12}^{(1,1)} ; & \widehat{f}_{\nu r}=\widehat{f}_{(\nu-r)} \longrightarrow \widehat{f} ; \\
\widehat{v}_{\nu r}=\widehat{v}_{(\nu-r)} \longrightarrow \widehat{v} &
\end{array}
$$

in equation (3.54). Then it can be rewritten as

$$
\left[\widehat{1}+(z-1) \widehat{f}^{2}-z(\widehat{1}-\widehat{v})\right] \widehat{\tilde{\varkappa}}_{\nu \mu}^{\prime}=\left[\widehat{1}-\widehat{f}^{2}\right] \delta_{\nu \mu}+\widehat{f} \cdot \widehat{v} \sum_{r=1}^{N} \pi_{\nu r} \cdot \widehat{\tilde{\varkappa}}_{r \mu}^{\prime},
$$

where

$$
\pi_{\nu r}= \begin{cases}1, & r \in \pi_{\nu} \\ 0, & r \notin \pi_{\nu}\end{cases}
$$

It should be remembered, that with putting $\varepsilon_{\nu}^{a}=0$ we go from the single-particle and two-particle intracluster CGF functionals (see (3.31), (3.32)) to the corresponding CGFs (see (3.36), (3.37)).

Going to the frequency-momentum representation in (3.56) and solving the obtained equation, we get for $\widehat{\tilde{\varkappa}}^{\prime}\left(\vec{q}, \omega_{n}\right)$

$$
\begin{aligned}
\widehat{\tilde{\varkappa}}^{\prime}\left(\vec{q}, \omega_{n}\right) & =\left[\widehat{1}+(z-1) \widehat{f}^{2}\left(\omega_{n}\right)-z\left[\widehat{1}-\widehat{v}\left(\omega_{n}\right)\right]-\widehat{f}\left(\omega_{n}\right) \cdot \widehat{v}\left(\omega_{n}\right) \cdot \pi(\vec{q})\right]^{-1} \\
& \times\left[\widehat{1}-\widehat{f}^{2}\left(\omega_{n}\right)\right] .
\end{aligned}
$$

Here

$$
\widehat{f}\left(\omega_{n}\right)=\left[\widehat{\mathcal{F}}_{12}^{(2,0)}\left(\omega_{n}\right)\right]^{-1} \cdot \widehat{\mathcal{F}}_{12}^{(1,1)}\left(\omega_{n}\right) ; \quad \widehat{v}\left(\omega_{n}\right)=\left[\widehat{\mathcal{F}}_{12}^{(2,0)}\left(\omega_{n}\right)\right]^{-1} \cdot \widehat{\mathcal{F}}_{1}^{(2)}\left(\omega_{n}\right)
$$

and $\widehat{\mathcal{F}}_{12}^{(2,0)}\left(\omega_{n}\right), \widehat{\mathcal{F}}_{12}^{(1,1)}\left(\omega_{n}\right), \widehat{\mathcal{F}}_{1}^{(2)}\left(\omega_{n}\right)$ are $3 \times 3$ matrices in the indices $a, b(a=x, y, z$ or,,$+- z)$, their elements are Fourier transforms $\left(\mathcal{F}_{12}^{(2,0)}\left(\begin{array}{l}a, b \\ \omega_{n}\end{array}\right), \mathcal{F}_{12}^{(1,1)}\left(\begin{array}{c}a, b \\ \omega_{n}\end{array}\right), \mathcal{F}_{1}^{(2)}\left(\begin{array}{l}a, b \\ \omega_{n}\end{array}\right)\right)$ of the pair intracluster CGFs $\left\langle T_{\tau} \overline{\bar{S}}_{1}^{a}(\tau) \overline{\bar{S}}_{1}^{b}(0)\right\rangle_{\rho_{12}}^{\mathrm{c}},\left\langle T_{\tau} \overline{\bar{S}}_{1}^{a}(\tau) \overline{\bar{S}}_{2}^{b}(0)\right\rangle_{\rho_{12}}^{\mathrm{c}},\left\langle T_{\tau} \bar{S}_{1}^{a}(\tau) \bar{S}_{1}^{b}(0)\right\rangle_{\rho_{1}}^{\mathrm{c}}$, respectively. $\pi(\vec{q})$ is the Fourier transform of the function $\pi_{\nu r}$. For simple lattices with a hypercubic symmetry, $\pi(\vec{q})$ reads

$$
\pi(\vec{q})=2 \sum_{i=1}^{d} \cos \left(q_{i} \cdot \alpha\right)
$$

$d$ is the lattice dimensionality; $\alpha$ is the lattice constant. The obtained matrix expression (3.58) can be rewritten as

$$
\begin{aligned}
\widehat{\varkappa}^{\prime}\left(\vec{q}, \omega_{n}\right) & =\left[z \widehat{v}\left(\omega_{n}\right)-(z-1)\left[\widehat{1}+\widehat{f}\left(\omega_{n}\right)\right]+z\left[\widehat{1}-\widehat{f}\left(\omega_{n}\right)\right]^{-1} \widehat{f}\left(\omega_{n}\right) \cdot \widehat{v}\left(\omega_{n}\right) \cdot \Theta(\vec{q})\right]^{-1} \\
& \times\left[\widehat{1}+\widehat{f}\left(\omega_{n}\right)\right]
\end{aligned}
$$


where $\Theta(\vec{q})$ for simple lattices with a hypercubic symmetry is

$$
\Theta(\vec{q})=1-\frac{\pi(\vec{q})}{z}=\frac{2}{d} \sum_{i=1}^{d} \sin ^{2}\left(\frac{q_{i} \cdot \alpha}{2}\right) .
$$

Putting $\varepsilon_{\nu}^{a}=0$, going to the uniform fields case in relation (3.45), and going to the frequency-momentum representation, we obtain expressions for pair CGFs, which are convenient to rewrite in a matrix form in the indices $a, b$ :

$$
{ }^{k} \widehat{b}^{(2)}\left(\vec{q}, \omega_{n}\right)=\widehat{\mathcal{F}}_{1}^{(2)}\left(\omega_{n}\right) \cdot \widehat{\tilde{\varkappa}}^{\prime}\left(\vec{q}, \omega_{n}\right) .
$$

Hence, in order to calculate the pair CGFs of the reference system in the uniform fields case from (3.63), we need to calculate (according to (3.61) and (3.59)) the single- and two-particle intracluster pair CGFs $\mathcal{F}_{1}^{(2)}\left(\begin{array}{c}a, b \\ \omega_{n}\end{array}\right)=\left(\left\langle T_{\tau} \bar{S}_{1}^{a}(\tau) \bar{S}_{1}^{b}(0)\right\rangle_{\rho_{1}}^{c}\right)_{\omega_{n}}$, $\mathcal{F}_{12}^{(2,0)}\left(\begin{array}{c}a, b \\ \omega_{n}\end{array}\right)=\left(\left\langle T_{\tau} \overline{\bar{S}}_{1}^{a}(\tau) \overline{\bar{S}}_{1}^{b}(0)\right\rangle_{\rho_{12}}^{\mathrm{c}}\right)_{\omega_{n}}, \mathcal{F}_{12}^{(1,1)}\left(\begin{array}{c}a, b \\ \omega_{n}\end{array}\right)=\left(\left\langle T_{\tau} \overline{\bar{S}}_{1}^{a}(\tau) \overline{\bar{S}}_{2}^{b}(0)\right\rangle_{\rho_{12}}^{c}\right)_{\omega_{n}}$.

\section{Ising model in transverse field}

\subsection{Thermodynamics. General results}

We consider the Ising model in transverse field with a renormalized pseudospin operator $\left(S^{z}=(-1,1)\right)$.

$$
H=-\sum_{\nu=1}^{N}\left(h S_{\nu}^{z}+\Gamma S_{\nu}^{x}\right)-\frac{1}{2} \sum_{\nu, \delta} K S_{\nu}^{z} S_{\nu+\delta}^{z}-\frac{1}{2} \sum_{\nu, \mu} J_{\nu \mu} S_{\nu}^{z} S_{\mu}^{z}
$$

Here $K$ and $J_{\nu \mu}$ are the short-range and long-range pair interactions, respectively; $\Gamma$ is the transverse field; the quantity $h \rightarrow 0$ is introduced for the sake of convenience. Hereafter, the factor $\beta=\left(k_{\mathrm{B}} T\right)^{-1}$ is written explicitly.

In the framework of MFA for the long-range interactions, the Hamiltonian (4.1) can be written as

$$
H={ }^{k} H+\frac{1}{2} N J_{0} m^{2}
$$

where

$$
J_{0}=\sum_{\mu=1}^{N} J_{\nu \mu}, \quad m=\left\langle S^{z}\right\rangle_{\rho}
$$

and ${ }^{k} H$ is the Hamiltonian of the reference IMTF

$$
\begin{aligned}
& { }^{k} H=-\sum_{\nu=1}^{N}\left[\varkappa^{z} S_{\nu}^{z}+\varkappa^{x} S_{\nu}^{x}\right]-\frac{1}{2} \sum_{\nu, \delta} K S_{\nu}^{z} S_{\nu+\delta}^{z} ; \\
& \varkappa^{z}=h+J_{0} m ; \quad \varkappa^{x}=\Gamma .
\end{aligned}
$$


According to the results of previous sections, the free energy of IMTF within the TPCA for the short-range interactions, with the long-range interactions taken into account within the MFA, is

$$
F=-k_{\mathrm{B}} T \cdot{ }^{k} \mathcal{F}+\frac{1}{2} N J_{0} m^{2}
$$

The ${ }^{k} \mathcal{F}$-function of the reference IMTF

$$
{ }^{k} \mathcal{F}=(1-z) N \mathcal{F}_{1}+\frac{z N}{2} \mathcal{F}_{12}
$$

is expressed via the single-particle

$$
\begin{aligned}
& \mathcal{F}_{1}=\ln Z_{1}, \quad Z_{1}=\operatorname{Sp}_{\boldsymbol{S}_{1}} \mathrm{e}^{-\beta H_{1}}, \\
& H_{1}=-\sum_{a=x, z} \tilde{\varkappa}^{a} S_{1}^{a} ; \quad \tilde{\varkappa}^{a}=\varkappa^{a}+z \varphi^{a}, \quad(a=z, x),
\end{aligned}
$$

and two-particle

$$
\begin{aligned}
& \mathcal{F}_{12}=\ln Z_{12}, \quad Z_{12}=\operatorname{Sp}_{\boldsymbol{S}_{1}, \boldsymbol{S}_{2}} \mathrm{e}^{-\beta H_{12}} ; \\
& H_{12}=-\sum_{a=x, z} \tilde{\tilde{\varkappa}}^{a}\left(S_{1}^{a}+S_{2}^{a}\right)-K S_{1}^{z} S_{2}^{z} ; \quad \tilde{\tilde{\varkappa}}^{a}=\varkappa^{a}+(z-1) \varphi^{a}, \quad(a=z, x)
\end{aligned}
$$

intracluster $\mathcal{F}$-functions. Let us show briefly how these functions can be obtained.

The Hamiltonian $H_{1}$ acts based on the two functions of state of a single particle

$$
\begin{array}{ll}
1 & + \\
2 & -
\end{array}
$$

In the representation (4.12), the single-particle Hamiltonian reads

$$
H_{1}=-\left(\begin{array}{cc}
\tilde{\varkappa}^{z} & \tilde{\varkappa}^{x} \\
\tilde{\varkappa}^{x} & -\tilde{\varkappa}^{z}
\end{array}\right)
$$

Taking into account (4.8), one can easily obtain the single-particle partition function in an explicit form

$$
Z_{1}=2 \operatorname{ch}(\beta \Lambda) ; \quad \Lambda=\sqrt{\left(\tilde{\varkappa}^{z}\right)^{2}+\left(\tilde{\varkappa}^{x}\right)^{2}}
$$

The two particle Hamiltonian $H_{12}$ acts based on the four functions of state of a two-particle cluster

$$
\begin{array}{lll}
1 & + & + \\
2 & + & - \\
3 & - & + \\
4 & - & -
\end{array}
$$


In the representation (4.15), the Hamiltonian $H_{12}$ reads

$$
H_{12}=-\left(\begin{array}{cccc}
2 \tilde{\tilde{\varkappa}}^{z}+K & \tilde{\tilde{\varkappa}}^{x} & \tilde{\tilde{\varkappa}}^{x} & 0 \\
\tilde{\tilde{\varkappa}}^{x} & -K & 0 & \tilde{\tilde{\varkappa}}^{x} \\
\tilde{\tilde{\varkappa}}^{x} & 0 & -K & \tilde{\tilde{\varkappa}}^{x} \\
0 & \tilde{\tilde{\varkappa}}^{x} & \tilde{\tilde{\varkappa}}^{x} & -2 \tilde{\tilde{\varkappa}}^{z}+K
\end{array}\right) .
$$

Based on (4.10) and (4.16) we obtain the two-particle partition function

$$
Z_{12}=\sum_{i=1}^{4} \mathrm{e}^{-\beta\left(E_{12}\right)_{i}}
$$

where

$$
\left(E_{12}\right)_{4}=K
$$

whereas three other eigenvalues $\left(E_{12}\right)_{1},\left(E_{12}\right)_{2},\left(E_{12}\right)_{3}$ of the matrix (4.16) are roots of a cubic equation

$$
E_{12}^{3}+K E_{12}^{2}-\left[K^{2}+4\left(\tilde{\tilde{\varkappa}}^{x}\right)^{2}+4\left(\tilde{\tilde{\varkappa}}^{z}\right)^{2}\right] E_{12}-K\left[K^{2}+4\left(\tilde{\tilde{\varkappa}}^{x}\right)^{2}-4\left(\tilde{\tilde{\varkappa}}^{z}\right)^{2}\right]=0 .
$$

From (3.43) and (3.44), taking into account the fact that in the framework of the MFA for the long-range interactions

$$
\left\langle S^{z}\right\rangle_{\rho}=-\frac{1}{N} \frac{\mathrm{d} F}{\mathrm{~d} h}={ }^{k}\left\langle S^{z}\right\rangle_{k_{\rho}}=-\frac{1}{N} \frac{\mathrm{d}{ }^{k} F}{\mathrm{~d} \varkappa^{z}}
$$

(this can be obtained from the explicit expression for the free energy (4.6)), we get equations for the parameters $m=\left\langle S^{z}\right\rangle_{\rho}, \eta=\left\langle S^{x}\right\rangle_{\rho}$ and cluster fields $\varphi^{a}(a=z, x)$

$$
\begin{aligned}
& \frac{\tilde{\varkappa}^{x}}{\Lambda} \operatorname{th}(\beta \Lambda)=\frac{4 \tilde{\tilde{\varkappa}}^{x}}{Z_{12}} \sum_{i=1}^{3} \frac{\left[-\left(E_{12}\right)_{i}-K\right] \mathrm{e}^{-\beta\left(E_{12}\right)_{i}}}{3\left(E_{12}\right)_{i}^{2}+2 K\left(E_{12}\right)_{i}-\left[K^{2}+4\left(\tilde{\tilde{\varkappa}}^{x}\right)^{2}+4\left(\tilde{\tilde{\varkappa}}^{z}\right)^{2}\right]}, \\
& \frac{\tilde{\varkappa}^{z}}{\Lambda} \operatorname{th}(\beta \Lambda)=\frac{4 \tilde{\tilde{\varkappa}}^{z}}{Z_{12}} \sum_{i=1}^{3} \frac{\left[-\left(E_{12}\right)_{i}+K\right] \mathrm{e}^{-\beta\left(E_{12}\right)_{i}}}{3\left(E_{12}\right)_{i}^{2}+2 K\left(E_{12}\right)_{i}-\left[K^{2}+4\left(\tilde{\tilde{\varkappa}}^{x}\right)^{2}+4\left(\tilde{\tilde{\varkappa}}^{z}\right)^{2}\right]}, \\
& m=\frac{\tilde{\varkappa}^{z}}{\Lambda} \operatorname{th}(\beta \Lambda), \\
& \eta=\frac{\tilde{\varkappa}^{x}}{\Lambda} \operatorname{th}(\beta \Lambda) .
\end{aligned}
$$

When the long-range interaction is absent $\left(J_{0}=0\right)$, we have a system of two equations (4.21) and (4.22) for $\varphi^{x}, \varphi^{z}$ in an implicit form $\left(\left(E_{12}\right)_{i}\right.$ are roots of cubic equation (4.19)) and expressions (4.23) for $m$ and (4.24) for $\eta$. When $J_{0} \neq 0$, we have a system of three equations $(4.21)-(4.23)$ for $\varphi^{x}, \varphi^{z}$, and $m$, and an expression for $\eta$. 
Numerical analysis of the thermodynamic characteristics and longitudinal static susceptibility $\chi^{z z}$ (which is too cumbersome to be presented here) obtained here within the TPCA in the short-range interactions, with the long-range interactions taken into account within the MFA, as well as the study of the applicability bounds of this approach to the IMTF on different types of lattices at different values of the parameters $\Gamma, J_{0}$ will be given elsewhere. Here we shall only briefly consider the major results at $K>0, J_{0} \geqslant 0, \Gamma>0$. We shall use the terminology of ferroelectricity.

For the one-dimensional IMTF at $J_{0}=0$, the two-particle cluster approximation, unlike the MFA for the short-range interactions, does not predict the existence of ferroelectric ordering (at $T>0$ and arbitrary $\Gamma$ the system is in the paraelectric phase). Comparison of the TPCA results for the free energy, entropy, and specific heat as functions of temperature (expressions for entropy and specific heat were obtained for the paraelectric phase only) at different values of $\Gamma / K$ has shown, that this approximation yields fair results for these characteristics at all temperatures except for the low-temperature region. Thus, at high temperatures, the TPCA results accord with exact results not only qualitatively, but also well enough quantitatively. The lower is the temperature the more the results of TPCA differ from the exact ones (too low values of free energy, entropy, and specific heat), whereas in the lowtemperature region $T<T_{1}\left(k_{\mathrm{B}} T_{1} / K<\operatorname{th}\left(\sqrt[4]{\frac{1}{6} \cdot \Gamma / K}\right)+\frac{1}{6} \cdot \Gamma / K\right)$ are qualitatively incorrect (for instance, the free energy is an increasing function of temperature).

For the one-dimensional model at $J_{0}>0$, as well as for two-dimensional and three-dimensional models at $J_{0} \geqslant 0$, the TPCA for the short-range interactions with the long-range interactions taken into account within the MFA predicts that a limiting value $(\Gamma / K)_{\mathrm{k}}$ exists which depends on $J_{0}$ and $z$, and above which a ferroelectric ordering is impossible (the latter is a qualitatively correct result). At $(\Gamma / K)_{\mathrm{a}}<$ $\Gamma / K<(\Gamma / K)_{\mathrm{k}}\left(\right.$ where $\left.(\Gamma / K)_{\mathrm{a}}=\sqrt{c\left(z, \Gamma, J_{0}, K\right) \cdot z J_{0} / K}, c\left(z, \Gamma, J_{0}, K\right) \approx 2\right)$ this approximation predicts a phase transition from the paraelectric phase to the ferroelectric phase on lowering temperature and the phase transition from the ferroelectric to the paraelectric phase - the so-called anti-Curie point. At small enough values of $\Gamma / K \leqslant(\Gamma / K)_{\mathrm{a}}$ the anti-Curie point is absent, but the temperature behaviour of the thermodynamic characteristics remains qualitatively incorrect. The low-temperature region of $T<T_{1}$, where the TPCA yields incorrect results for thermodynamic characteristics, is reduced when the values of $\Gamma / K$ and $J_{0} / K$ decrease (see table 1 ). In the high-temperature region $T>T_{1}$ at $J_{0}=0$ and $z>2$, the TPCA is much more correct than the mean field approximation for the short-range interactions.

We also performed a numerical analysis of the TPCA results at neglecting the variational parameter $\varphi^{x}\left(\varphi^{x}=0\right)$. This version of the approximation is not suitable for one-dimensional chains. Thus, at small enough values of $J_{0} / K<0.09$ and $\Gamma / K$, Curie temperature increases on increasing $\Gamma / K$. At $J_{0}=0$ and $\left.\left.\Gamma / K \in\right] 0,1.28\right]$ a ferroelectric ordering is predicted.

For two-dimensional and three-dimensional lattices, neglecting the variational parameter $\varphi^{x}$ leads to a slight quantitative worsening of the results in a hightemperature region and to a qualitatively correct description of temperature dependences of thermodynamic characteristics $\left(m(T), \chi^{z z}(T)\right)$ in a low-temperature 
Table 1. Temperature of the anti-Curie point $T_{\mathrm{a}}$, temperature $T_{1}$ below which unphysical results for $m(T)$ and $\chi^{z z}(T)$ are obtained, and Curie temperature for a square lattice $(z=4)$ at different values of $\Gamma$ and $J_{0}$ within the TPCA for the short-range interactions, calculated with the long-range interactions taken into account in the MFA.

\begin{tabular}{|l|l|l|l|l|}
\hline$k_{\mathrm{B}} T_{\mathrm{a}} / K$ & $k_{\mathrm{B}} T_{\mathrm{l}} / K$ & $k_{\mathrm{B}} T_{\mathrm{c}} / K$ & $J_{0} / K$ & $\Gamma / K$ \\
\hline 0.01 & 0.57 & 2.86 & 0.0 & 0.5 \\
0.26 & 0.93 & 2.40 & 0.0 & 2.0 \\
0.03 & 0.96 & 3.04 & 0.4 & 2.0 \\
\hline
\end{tabular}

region. The larger is the lattice dimensionality and the value of the long-range interaction and the smaller is the transverse field the smaller is the mentioned worsening.

\subsection{Thermodynamics and intracluster pair distribution functions in para- electric phase}

In order to study the dynamic characterictics of the IMTF in the paraelectric phase, we write here certain relations for some thermodynamic quantities in the paraelectric phase $\left(\varkappa^{z}=\tilde{\varkappa}^{z}=\tilde{\varkappa}^{z}=\varphi^{z}=0, m=0\right)$. The eigenvalues $\left(E_{12}\right)_{i}$ of two-particle Hamiltonian (4.16) in the paraelectric phase are (see (4.19)):

$$
\left(E_{12}\right)_{1}=-L ; \quad\left(E_{12}\right)_{2}=L ; \quad\left(E_{12}\right)_{3}=K ; \quad\left(E_{12}\right)_{4}=-K
$$

where

$$
L=\sqrt{K^{2}+4\left(\tilde{\tilde{\varkappa}}^{x}\right)^{2}}
$$

From (4.25) we obtain the two-particle partition function in the paraelectric phase explicitly

$$
Z_{12}=2[\operatorname{ch}(\beta L)+\operatorname{ch}(\beta K)]
$$

In the paraelectric phase, equations (4.22), (4.23) turn to identity, while equation (4.21) for the variational parameter $\varphi^{x}$ can be written explicitly, using (4.25)

$$
\operatorname{th}\left(\beta \tilde{\varkappa}^{x}\right)=\frac{4 \tilde{\tilde{\varkappa}}^{x}}{L Z_{12}} \operatorname{sh}(\beta L) .
$$

We also present here an expression for $\eta=\left\langle S^{x}\right\rangle_{\rho}$ :

$$
\eta=\operatorname{th}\left(\beta \tilde{\varkappa}^{x}\right) .
$$

To calculate the pair CGFs of the reference model (4.4) within the TPCA (see (3.63)), we need to know the single-particle and two-particle intracluster pair CGFs $\mathcal{F}_{1}^{(2)}\left(\begin{array}{c}a, b \\ \omega_{n}\end{array}\right), \mathcal{F}_{12}^{(2,0)}\left(\begin{array}{l}a, b \\ \omega_{n}\end{array}\right), \mathcal{F}_{12}^{(1,1)}\left(\begin{array}{c}a, b \\ \omega_{n}\end{array}\right)(a, b=x, y, z)$. Let us calculate now the two-particle 
intracluster CGFs. It is convenient to do so in the self-representation of the operator $H_{12}$.

Since we have explicit expressions for the eigenvalues of the two-particle Hamiltonian (4.16) in the paraelectric phase (see (4.25)), it is easy to obtain the normalized unitary matrix, which diagonalizes the two-particle Hamiltonian

$$
\hat{U}=\left(\begin{array}{cccc}
r_{1} & r_{2} & 0 & 1 / \sqrt{2} \\
r_{2} & -r_{1} & 1 / \sqrt{2} & 0 \\
r_{2} & -r_{1} & -1 / \sqrt{2} & 0 \\
r_{1} & r_{2} & 0 & -1 / \sqrt{2}
\end{array}\right) .
$$

Here we use the notations

$$
r_{1}=\frac{1}{2} \cdot \sqrt{1+K / L} ; \quad r_{2}=\frac{1}{2} \cdot \sqrt{1-K / L} .
$$

Going from the Pauli operators to their four-row analogs [50-52]

$$
\sigma_{1}^{a}=S_{1}^{a} \otimes I ; \quad \sigma_{2}^{a}=I \otimes S_{2}^{a} ; \quad a=x, y, z
$$

(here $I$ is the two-row unit matrix, $\otimes$ is the direct product symbol; matrices $\sigma_{\nu}^{a}$ obey Pauli commutation rules) and performing a unitary transformation

$$
\tilde{\sigma}_{\nu}^{a}=\hat{U}^{-1} \sigma_{\nu}^{a} \hat{U}
$$

we obtain the pseudospin operators in the self-representation of the operator $H_{12}$ :

$$
\begin{aligned}
& \tilde{\sigma}_{1}^{z}=\sqrt{2}\left(\begin{array}{cccc}
0 & 0 & r_{2} & r_{1} \\
0 & 0 & -r_{1} & r_{2} \\
r_{2} & -r_{1} & 0 & 0 \\
r_{1} & r_{2} & 0 & 0
\end{array}\right) ; \quad \tilde{\sigma}_{2}^{z}=\sqrt{2}\left(\begin{array}{cccc}
0 & 0 & -r_{2} & r_{1} \\
0 & 0 & r_{1} & r_{2} \\
-r_{2} & r_{1} & 0 & 0 \\
r_{1} & r_{2} & 0 & 0
\end{array}\right) ; \\
& \tilde{\sigma}_{1}^{x}=\left(\begin{array}{cccc}
2 \tilde{\tilde{\varkappa}}^{x} / L & -K / L & 0 & 0 \\
-K / L & -2 \tilde{\tilde{\varkappa}}^{x} / L & 0 & 0 \\
0 & 0 & 0 & -1 \\
0 & 0 & -1 & 0
\end{array}\right) ; \quad \tilde{\sigma}_{2}^{x}=\left(\begin{array}{cccc}
2 \tilde{\tilde{\varkappa}}^{x} / L & -K / L & 0 & 0 \\
-K / L & -2 \tilde{\tilde{\varkappa}}^{x} / L & 0 & 0 \\
0 & 0 & 0 & 1 \\
0 & 0 & 1 & 0
\end{array}\right) ; \\
& \tilde{\sigma}_{1}^{y}=\mathrm{i} \sqrt{2}\left(\begin{array}{cccc}
0 & 0 & r_{1} & r_{2} \\
0 & 0 & r_{2} & -r_{1} \\
-r_{1} & -r_{2} & 0 & 0 \\
-r_{2} & r_{1} & 0 & 0
\end{array}\right) ; \quad \tilde{\sigma}_{2}^{y}=\mathrm{i} \sqrt{2}\left(\begin{array}{cccc}
0 & 0 & -r_{1} & r_{2} \\
0 & 0 & -r_{2} & -r_{1} \\
r_{1} & r_{2} & 0 & 0 \\
-r_{2} & r_{1} & 0 & 0
\end{array}\right) .
\end{aligned}
$$

Expanding operators $\tilde{\sigma}_{i}^{a}$ (4.34) in finite series in the four-dimensional Hubbard operators [50-52], following [14,57], we easily calculate the two-particle cumulant pair intracluster Green functions:

$$
\begin{aligned}
& \widehat{\mathcal{F}}_{12}^{(2,0)}\left(\omega_{n}\right)=\left(\begin{array}{ccc}
\mathcal{F}_{12}^{(2,0)}\left(\begin{array}{l}
x, x \\
\omega_{n}
\end{array}\right) & 0 & 0 \\
0 & \mathcal{F}_{12}^{(2,0)}\left(\begin{array}{c}
y, y \\
\omega_{n}
\end{array}\right) & \mathcal{F}_{12}^{(2,0)}\left(\begin{array}{c}
y, z \\
\omega_{n}
\end{array}\right) \\
0 & \mathcal{F}_{12}^{(2,0)}\left(\begin{array}{c}
z, y \\
\omega_{n}
\end{array}\right) & \mathcal{F}_{12}^{(2,0)}\left(\begin{array}{c}
(z, z \\
\omega_{n}
\end{array}\right)
\end{array}\right), \\
& \widehat{\mathcal{F}}_{12}^{(1,1)}\left(\omega_{n}\right)=\left(\begin{array}{ccc}
\mathcal{F}_{12}^{(1,1)}\left(\begin{array}{c}
x, x \\
\omega_{n}
\end{array}\right) & 0 & 0 \\
0 & \mathcal{F}_{12}^{(1,1)}\left(\begin{array}{c}
y, y \\
\omega_{n}
\end{array}\right) & \mathcal{F}_{12}^{(1,1)}\left(\begin{array}{c}
y, z \\
\omega_{n}
\end{array}\right) \\
0 & \mathcal{F}_{12}^{(1,1)}\left(\begin{array}{c}
z, y \\
\omega_{n}
\end{array}\right) & \mathcal{F}_{12}^{(1,1)}\left(\begin{array}{c}
z, z \\
\omega_{n}
\end{array}\right)
\end{array}\right),
\end{aligned}
$$


where

$$
\begin{aligned}
& \mathcal{F}_{12}^{(2,0)}\left(\begin{array}{l}
x, x \\
\omega_{n}
\end{array}\right)=\left(\left\langle T_{\tau} \tilde{\sigma}_{1}^{x}(\tau) \tilde{\sigma}_{1}^{x}(0)\right\rangle_{\rho_{12}}^{\mathrm{c}}\right)_{\omega_{n}}=A_{s} \delta\left(\omega_{n}\right)+A_{+}\left(\omega_{n}\right) ; \\
& \mathcal{F}_{12}^{(2,0)}\left(\begin{array}{c}
y, y \\
\omega_{n}
\end{array}\right)=\frac{4}{\beta L Z_{12} \psi\left(\omega_{n}\right)}\left[\left(2 \tilde{\tilde{\varkappa}}^{x}\right)^{4} \operatorname{sh}(\beta L)+C_{+} \cdot \omega_{n}^{2}\right] ; \\
& \mathcal{F}_{12}^{(2,0)}\left(\begin{array}{c}
y, z \\
\omega_{n}
\end{array}\right)=-\mathcal{F}_{12}^{(2,0)}\left(\begin{array}{c}
z, y \\
\omega_{n}
\end{array}\right)=\frac{8 \tilde{\tilde{\varkappa}}^{x} \omega_{n}}{\beta L Z_{12} \psi\left(\omega_{n}\right)}\left[C_{-}+\operatorname{sh}(\beta L) \omega_{n}^{2}\right] ; \\
& \mathcal{F}_{12}^{(2,0)}\left(\begin{array}{l}
z, z \\
\omega_{n}
\end{array}\right)=\frac{16\left(\tilde{\tilde{\varkappa}}^{x}\right)^{2}}{\beta L Z_{12} \psi\left(\omega_{n}\right)}\left[C_{-}+\operatorname{sh}(\beta L) \omega_{n}^{2}\right] \\
& \mathcal{F}_{12}^{(1,1)}\left(\begin{array}{l}
x, x \\
\omega_{n}
\end{array}\right)=\left(\left\langle T_{\tau} \tilde{\sigma}_{1}^{x}(\tau) \tilde{\sigma}_{2}^{x}(0)\right\rangle_{\rho_{12}}^{\mathrm{c}}\right)_{\omega_{n}}=A_{s} \delta\left(\omega_{n}\right)+A_{-}\left(\omega_{n}\right) ; \\
& \mathcal{F}_{12}^{(1,1)}\left(\begin{array}{c}
y, y \\
\omega_{n}
\end{array}\right)=-\frac{16 B K \omega_{n}^{2}}{\beta Z_{12} \psi\left(\omega_{n}\right)} \\
& \mathcal{F}_{12}^{(1,1)}\left(\begin{array}{c}
y, z \\
\omega_{n}
\end{array}\right)=-\mathcal{F}_{12}^{(1,1)}\left(\begin{array}{c}
z, y \\
\omega_{n}
\end{array}\right)=\frac{32 B K \tilde{\tilde{\varkappa}}^{x} \omega_{n}}{\beta Z_{12} \psi\left(\omega_{n}\right)} ; \\
& \mathcal{F}_{12}^{(1,1)}\left(\begin{array}{c}
z, z \\
\omega_{n}
\end{array}\right)=\frac{64 B K\left(\tilde{\tilde{\varkappa}}^{x}\right)^{2}}{\beta Z_{12} \psi\left(\omega_{n}\right)} \text {. }
\end{aligned}
$$

Here we use the notations

$$
\begin{aligned}
& A_{s}=\left(\frac{4 \tilde{\tilde{\varkappa}}^{x}}{L Z_{12}}\right)^{2} \cdot[1+\operatorname{ch}(\beta K) \operatorname{ch}(\beta L)] ; \quad B=\frac{1}{2}[\operatorname{ch}(\beta L)-\operatorname{ch}(\beta K)] ; \\
& A_{ \pm}\left(\omega_{n}\right)=\frac{8 K}{\beta L Z_{12}}\left(\frac{K \operatorname{sh}(\beta L)}{4 L^{2}+\omega_{n}^{2}} \pm \frac{L \operatorname{sh}(\beta K)}{4 K^{2}+\omega_{n}^{2}}\right) ; \\
& C_{ \pm}=\left[L^{2}+K^{2}\right] \operatorname{sh}(\beta L) \pm 2 L K \operatorname{sh}(\beta K) ; \\
& \psi\left(\omega_{n}\right)=\left[(L+K)^{2}+\omega_{n}^{2}\right]\left[(L-K)^{2}+\omega_{n}^{2}\right] .
\end{aligned}
$$

The most convenient way of obtaining the single-particle intracluster CGFs is, by performing a rotation in a spin space, to go to such a coordinate system, where Hamiltonian (4.13) is diagonal. We present here the final result (after the inverse transformation) in the paraelectric phase in terms of $a=x, y, z$ :

$$
\widehat{\mathcal{F}}_{1}^{(2)}\left(\omega_{n}\right)=\left(\begin{array}{ccc}
{\left[1-\eta^{2}\right] \delta\left(\omega_{n}\right)} & 0 & 0 \\
0 & g\left(\omega_{n}\right) & g^{\prime}\left(\omega_{n}\right) \\
0 & -g^{\prime}\left(\omega_{n}\right) & g\left(\omega_{n}\right)
\end{array}\right) .
$$

Here we use notations

$$
g\left(\omega_{n}\right)=\frac{4}{\beta} \cdot \frac{\eta \cdot \tilde{\varkappa}^{x}}{\left(2 \tilde{\varkappa}^{x}\right)^{2}+\omega_{n}^{2}} ; \quad g^{\prime}\left(\omega_{n}\right)=\frac{\omega_{n}}{2 \tilde{\varkappa}^{x}} \cdot g\left(\omega_{n}\right) .
$$

At obtaining (4.40) we used the fact that within TPCA for the short-range interactions, taking into account the long-range interactions in the MFA, $\left\langle S^{x}\right\rangle_{\rho_{1}}=\left\langle S^{x}\right\rangle_{\rho} \equiv$ $\eta$. 


\subsection{Dynamics in paraelectric phase. Cluster random phase approximation}

Our task is to investigate the dynamics characteristics of the IMTF in the paraelectric phase within the TPCA for the short-range interactions and within the $\left(r_{0}^{-d}\right)^{0}$ approximation for the long-range interactions $[54,58]$ - the cluster random phase approximation. The first step is then to calculate the temperature CGFs.

In CRPA the pair CGF $\widehat{\mathcal{G}}\left(\vec{q}, \omega_{n}\right) \equiv \widehat{b}^{(2)}\left(\vec{q}, \omega_{n}\right)$ according to $(2.14)$ is

$$
\widehat{\mathcal{G}}\left(\vec{q}, \omega_{n}\right)=\left[1-{ }^{k} \widehat{\mathcal{G}}\left(\vec{q}, \omega_{n}\right) \beta \hat{J}(\vec{q})\right]^{-1} \widehat{\widehat{\mathcal{G}}}\left(\vec{q}, \omega_{n}\right),
$$

where

$$
\hat{J}(\vec{q})=\left(\begin{array}{ccc}
0 & 0 & 0 \\
0 & 0 & 0 \\
0 & 0 & J(\vec{q})
\end{array}\right),
$$

(in terms of $a=x, y, z)$, and ${ }^{k} \widehat{\mathcal{G}}\left(\vec{q}, \omega_{n}\right) \equiv{ }^{k} \widehat{b}^{(2)}\left(\vec{q}, \omega_{n}\right)$ is the pair CGF of the reference system, which in the TPCA reads (3.63).

From (4.42), (3.63), using (4.35), (4.40), we obtain pair CGFs. For $\mathcal{G}^{x x}\left(\vec{q}, \omega_{n}\right)$, and $\mathcal{G}^{z z}\left(\vec{q}, \omega_{n}\right)$ in the paraelectric phase we have:

$$
\mathcal{G}^{x x}\left(\vec{q}, \omega_{n}\right)=\delta\left(\omega_{n}\right) \cdot \mathcal{G}_{\alpha}^{x x}(\vec{q}), \quad \mathcal{G}^{z z}\left(\vec{q}, \omega_{n}\right)=\frac{4 \Gamma \eta\left[p_{+}+\omega_{n}^{2}\right]\left[p_{-}+\omega_{n}^{2}\right]}{R\left(\vec{q}, \omega_{n}\right)}
$$

Here we introduce the notations

$$
\begin{aligned}
& \mathcal{G}_{\alpha}^{x x}(\vec{q})=\left[1-\eta^{2}\right]\left\{\frac{z\left[1-\eta^{2}\right]}{d_{x}(T)}-(z-1)+z\left[1-\eta^{2}\right] \frac{b_{x}(T)}{d_{x}(T)} \Theta(\vec{q})\right\}^{-1} ; \\
& d_{x}(T)=\frac{K^{2}}{L^{2}} \cdot \frac{\eta}{\beta \tilde{\tilde{\varkappa}}^{x}}+2\left[1-\frac{K^{2}}{L^{2}}\right] \frac{1+\operatorname{ch}(\beta L) \operatorname{ch}(\beta K)}{\operatorname{ch}(\beta L)+\operatorname{ch}(\beta K)} ; \\
& b_{x}(T)=\frac{1}{2 \operatorname{sh}(\beta K)}\left\{\frac{K^{3}}{L^{3}} \operatorname{sh}(\beta L)-\operatorname{sh}(\beta K)+\beta K\left[1-\frac{K^{2}}{L^{2}}\right] \frac{1+\operatorname{ch}(\beta K) \operatorname{ch}(\beta L)}{\operatorname{ch}(\beta L)+\operatorname{ch}(\beta K)}\right\} ; \\
& R\left(\vec{q}, \omega_{n}\right)=\left[p_{-}+\omega_{n}^{2}\right]\left[\omega_{n}^{4}+\mathrm{u}_{2} \omega_{n}^{2}+\mathrm{u}_{0}-4 \Gamma \eta J(\vec{q})\left[p_{+}+\omega_{n}^{2}\right]\right] \\
& \quad+\frac{4 B L \Gamma K}{\tilde{\tilde{\varkappa}}^{x} \operatorname{sh}(\beta L)} \cdot \psi\left(\omega_{n}\right) z \Theta(\vec{q}) ; \\
& p_{ \pm}=K^{2}+L^{2}+\frac{2 L K[-\operatorname{sh}(\beta K) \pm 2 B]}{\operatorname{sh}(\beta L)} ; \quad p_{s}=K^{2}+L^{2}+\frac{2 L K[\operatorname{sh}(\beta K)-2 B]}{\operatorname{sh}(\beta L)} \\
& \mathrm{u}_{2}=2 z^{2}\left[K^{2}+L^{2}\right]+(z-1)^{2}\left[\left(2 \tilde{\varkappa}^{x}\right)^{2}+p_{+}\right]-2 z(z-1)\left\{\frac{\tilde{\varkappa}^{x}\left[\left(2 \tilde{\tilde{\varkappa}}^{x}\right)^{2}+p_{s}\right]}{2 \tilde{\tilde{\varkappa}}^{x}}+p_{+}\right\} \\
& \mathrm{u}_{0}=4 \Gamma\left\{4 z\left(\tilde{\tilde{\varkappa}}^{x}\right)^{3}-(z-1) \tilde{\varkappa}^{x} p_{+}\right\}
\end{aligned}
$$

In calculations of (4.44) we used the relations (4.28), (4.29). 
It should be noted that from (4.44) one obtains the static longitudinal susceptibility $\left(\chi^{z z}=\beta \mathcal{G}^{z z}(0,0)\right)$ of the IMTF in the paraelectric phase

$$
\chi^{z z}=\left[\frac{z L Z_{12}}{2\left\{\frac{L+K}{L-K} \cdot \mathrm{e}^{\beta L}-\frac{L-K}{L+K} \cdot \mathrm{e}^{-\beta L}-\frac{4 L K}{L^{2}-K^{2}} \cdot \mathrm{e}^{\beta K}\right\}}-\frac{(z-1) \tilde{\varkappa}^{x}}{\operatorname{th}\left(\beta \tilde{\varkappa}^{x}\right)}-J_{0}\right]^{-1},
$$

$\left(J_{0}=J(\vec{q}=0)\right)$ which accords with the one calculated from thermodynamic relations in the TPCA for the short-range interactions, with the long-range interactions taken into account in the MFA.

To explore the dynamic properties of the IMTF we needn't know the temperature CGFs (4.44), but the retarded CGFs. We can calculate them [59] by performing analytical continuation of the temperature CGFs $\mathcal{G}^{a b}\left(\vec{q}, \omega_{n}\right)\left(\mathrm{i} \omega_{n} \rightarrow E+\mathrm{i} E^{\prime}\right)$ and going to the limit $E^{\prime} \rightarrow 0$. The final results for spectral densities $\mathcal{J}^{x x}(\vec{q}, E)$ and $\mathcal{J}^{z z}(\vec{q}, E)$, defined as

$$
\mathcal{J}^{a b}(\vec{q}, E)=\lim _{E^{\prime} \rightarrow 0}\left[\frac{2 \hbar \beta}{\mathrm{e}^{\beta E}-1} \operatorname{Im} \mathcal{G}^{a b}\left(\vec{q}, \omega_{n}\right)_{\omega_{n} \rightarrow-\mathrm{i} E+E^{\prime}}\right]
$$

and for the pair cumulant correlation function $\left\langle S_{\vec{q}}^{z} S_{-\vec{q}}^{z}\right\rangle^{\mathrm{c}}$ are the following.

The spectral density $\mathcal{J}^{x x}(\vec{q}, E)$ of the IMTF in the paraelectric phase within the CRPA reads

$$
\mathcal{J}^{x x}(\vec{q}, E)=\delta(E) \cdot \mathcal{G}_{\alpha}^{x x}(\vec{q})
$$

Let us note, that an exact expression for $\mathcal{J}^{x x}(\vec{q}, E)$ of the one-dimensional IMTF with the short-range interactions only [31] has not only the central peak $(\sim \delta(E))$ but also two symmetrical resonance zones. The absence of the resonance zones within the CRPA for $\mathcal{J}^{x x}(\vec{q}, E)$ results from neglecting the fluctuations of cluster fields in this approximation.

The spectral density $\mathcal{J}^{z z}(\vec{q}, E)$ (in the paraelectric phase) can be presented as

$$
\frac{1}{\hbar} \mathcal{J}^{z z}(\vec{q}, E)=\sum_{i=-,+, r} k_{i}^{J}(\vec{q})\left[\delta\left(E-E_{i}(\vec{q})\right)+\mathrm{e}^{\beta E_{i}(\vec{q})} \delta\left(E+E_{i}(\vec{q})\right)\right]
$$

where $E_{i}(\vec{q})(i=-,+, r)$ are the elementary excitation spectrum modes, determined from the equation (see (4.44), (4.46))

$$
R\left(\vec{q}, \omega_{n}\right)_{\omega_{\omega_{n} \rightarrow-\mathrm{i} E}}=0
$$

and $k_{i}^{J}(\vec{q})$ are the integral intensities of the elementary excitations spectrum modes

$$
k_{i}^{J}(\vec{q})=4 \pi T \Gamma \eta \mathcal{A}_{i}(\vec{q}) \cdot \frac{\left[p_{+}-E_{i}^{2}(\vec{q})\right]\left[p_{-}-E_{i}^{2}(\vec{q})\right]}{\left(\mathrm{e}^{\beta E_{i}(\vec{q})}-1\right) E_{i}(\vec{q})} .
$$

Here we use the notations:

$$
\mathcal{A}_{i_{1}}(\vec{q})=\frac{1}{\left[E_{i_{1}}^{2}(\vec{q})-E_{i_{2}}^{2}(\vec{q})\right]\left[E_{i_{1}}^{2}(\vec{q})-E_{i_{3}}^{2}(\vec{q})\right]}, \quad \begin{aligned}
& i_{1}, i_{2}, i_{3}=(-,+, r), \\
& i_{1} \neq i_{2}, \quad i_{2} \neq i_{3}, \quad i_{3} \neq i_{1} .
\end{aligned}
$$


It should be noted that at $\vec{q}=0$ (see (4.44), (4.46)) there are only two modes

$$
E_{ \pm}(0)=\frac{1}{\sqrt{2}} \sqrt{\mathrm{U}_{2} \pm \sqrt{\mathrm{U}_{2}^{2}-4 \mathrm{U}_{0}}}
$$

where

$$
\mathrm{U}_{2}=\mathrm{u}_{2}-4 \eta \Gamma J_{0} ; \quad \mathrm{U}_{0}=\mathrm{u}_{0}-4 \eta \Gamma J_{0} p_{+} .
$$

The mode $E_{-}(\vec{q})$ is soft $\left(E_{-}(0) \rightarrow 0, T \rightarrow T_{\mathrm{c}}\right)$.

For the pair cumulant correlation function $\left\langle S_{\vec{q}}^{z} S_{-\vec{q}}^{z}\right\rangle^{c}$ from

$$
\left\langle S_{\vec{q}}^{a} S_{-\vec{q}}^{b}\right\rangle_{\left.\right|_{t \rightarrow 0} ^{c}}=\frac{1}{\hbar} \int_{-\infty}^{\infty} \mathrm{d} E \mathcal{J}^{a b}(\vec{q}, E)
$$

we obtain

$$
\left.\left\langle S_{\vec{q}}^{z} S_{-\vec{q}}^{z}\right\rangle^{c}\right|_{t \longrightarrow 0}=2 T \Gamma \eta \sum_{i=-,+, r} \mathcal{A}_{i}(\vec{q}) \cdot \operatorname{cth}\left(\frac{1}{2} \beta E_{i}\right) \cdot \frac{\left[p_{+}-E_{i}^{2}(\vec{q})\right]\left[p_{-}-E_{i}^{2}(\vec{q})\right]}{E_{i}(\vec{q})} .
$$

Let us briefly consider the results of numerical analysis of the longitudinal characteristics of the IMTF at $z=2, J_{\nu \mu}=0(K=1)$. As we have already mentioned, within CRPA the spectrum of the longitudinal characteristics of the model contains three nondamping modes $E_{-}(q), E_{+}(q), E_{r}(q)$ with the integral intensities $k_{-}^{J}(q)$, $k_{+}^{J}(q)$ and $k_{r}^{J}(q)$ in the spectral density $\mathcal{J}^{z z}(q, E)$. The calculated dependences of $E_{i}(q), k_{i}^{J}(q)$ at different transverse fields and temperatures are presented in figure 1. On increasing $\Gamma$, temperature, and $q$, redistribution of the intensities from low frequencies to higher frequencies is observed. At large $\Gamma$ and at low temperatures the redistribution on increasing $q$ takes place, first, mainly from $E_{-}(q)$ to $E_{r}(q)$, and then from $E_{r}(q)$ to $E_{+}(q)$. At large $\Gamma$ and at high temperatures the redistribution takes place mainly from $E_{-}(q)$ to $E_{+}(q)$. At small $\Gamma$ the redistribution is practically absent.

In figure 2 we present exact and approximate (CRPA) results for the static correlator $\left\langle S_{\nu}^{z} S_{\nu+n}^{z}\right\rangle$ at $k_{\mathrm{B}} T=0.6$ and $k_{\mathrm{B}} T=1.0$ at different $\Gamma$. The CRPA gives too low values of $\left\langle S_{\nu}^{z} S_{\nu+n}^{z}\right\rangle$, especially at low temperatures. Thus, the autocorrelator $\left\langle S_{\nu}^{z} S_{\nu}^{z}\right\rangle$ at low temperatures is essentially smaller than unity. The higher is the temperature and the smaller is $\Gamma$ the better the CRPA results accord with the exact ones.

Let us also discuss the redistribution of the modes intensities $E_{-}(0), E_{+}(0)$ on changing $\Gamma$ and temperature. At temperatures $k_{\mathrm{B}} T>\sqrt{\Gamma}$, change of the modes positions and integral intensities (obtained within the CRPA) on changing $\Gamma$ and $T$ qualitatively describes the change in the frequency dependences of the real part of the relaxation function $\operatorname{Re} \Psi^{z z}(0, E)$, calculated numerically $[35,36]$ or exactly. Thus, for instance, at $\Gamma=1$ and $k_{\mathrm{B}} T=0.8$ (see figure 3 ) $\operatorname{Re} \Psi^{z z}(0, E)$ has a prominent resonance zone at $E$ close to zero. Smearing of this resonance zone on increasing $T$ is qualitatively described by increasing $k_{+}^{J}(0)$ and $E_{-}(0)$ and by decreasing $k_{-}^{J}(0)$. On the other hand, for instance, at $T \rightarrow \infty$ (see figure 4 ), a shift of the resonance zone $\operatorname{Re} \Psi^{z z}(0, E)$ to higher frequencies region on increasing $\Gamma$ is described by increasing 

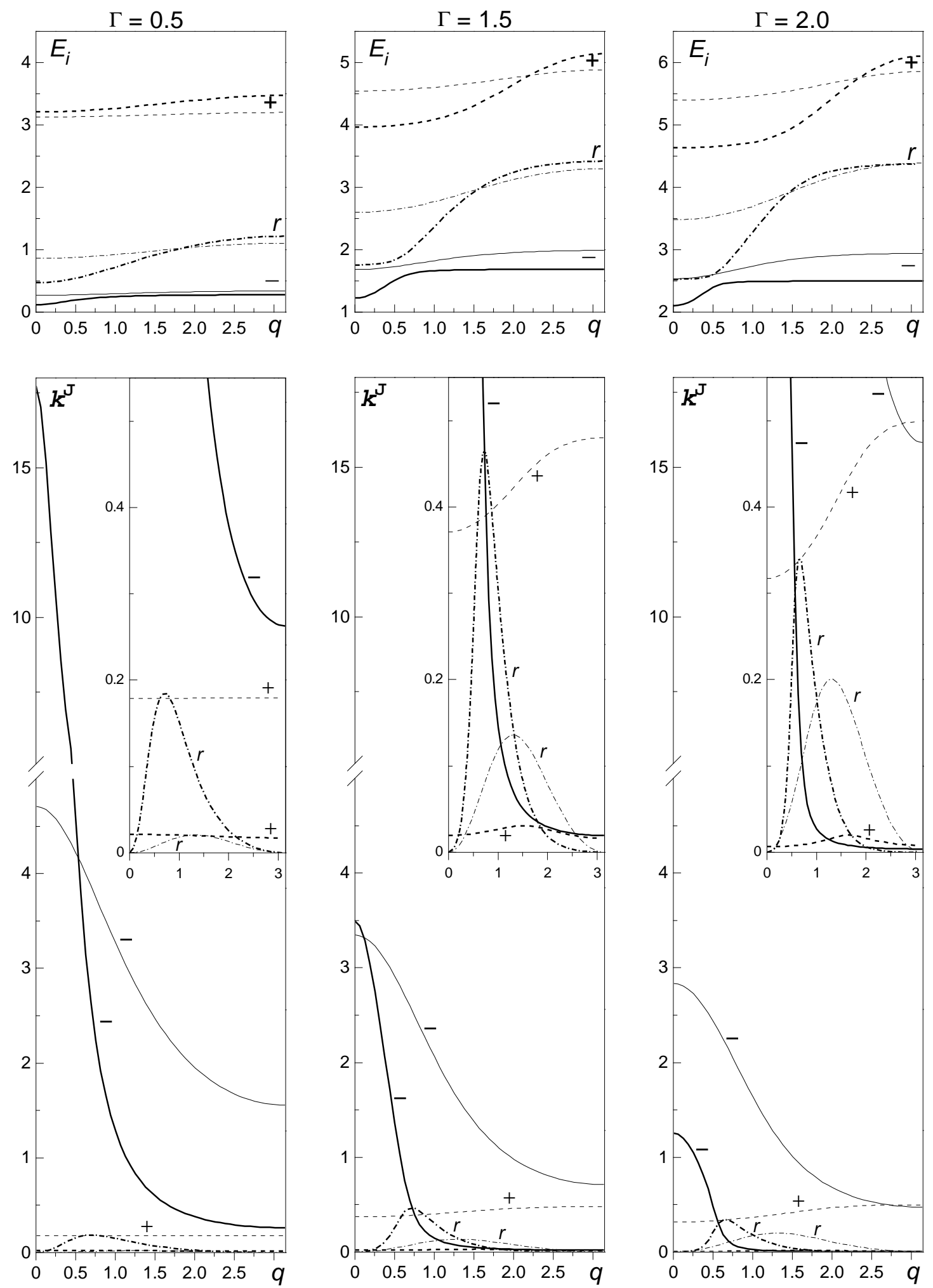

Figure 1. Elementary excitations spectrum modes $E_{i}(q)$ and their integral intensities $k_{i}^{J}(q)$ (within CRPA) as functions of quasimomentum $q$ at different temperatures (thick lines $-k_{\mathrm{B}} T=1.0$, thin lines $-k_{\mathrm{B}} T=4.0$ ) for $\Gamma=0.5,1.5,2.0$. 

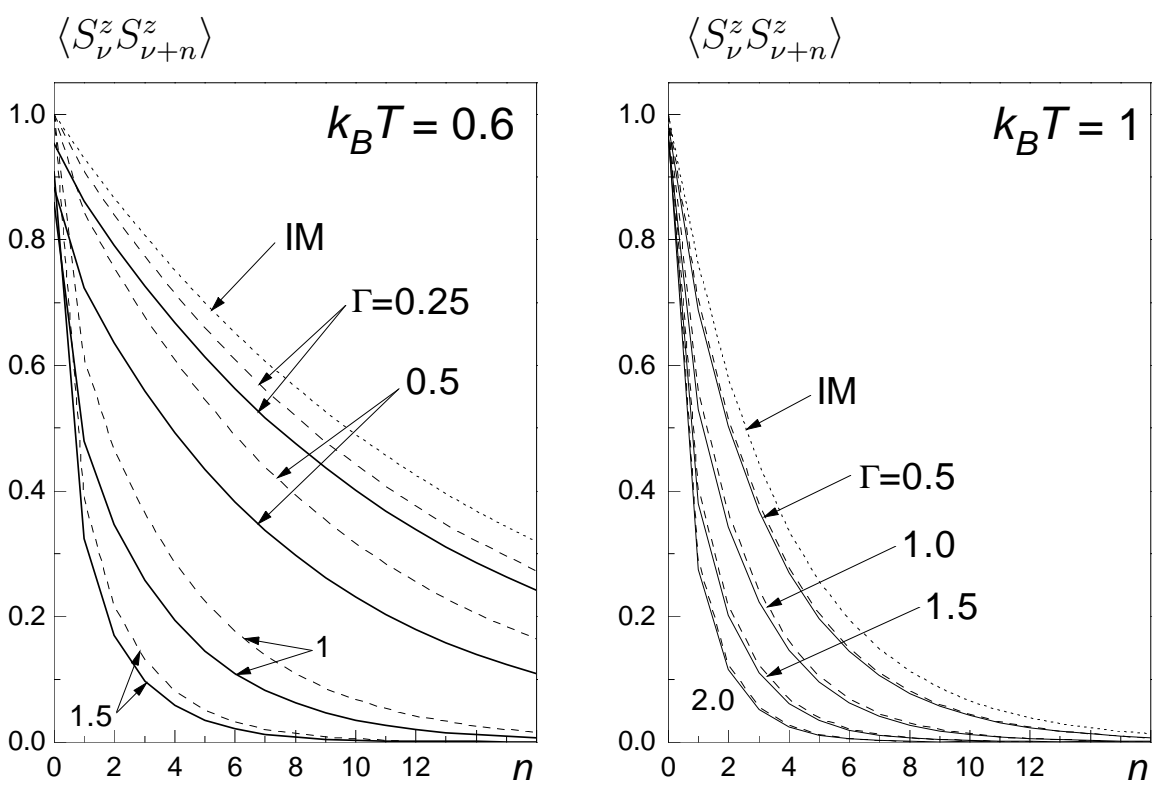

Figure 2. Static correlation function $\left\langle S_{\nu}^{z} S_{\nu+n}^{z}\right\rangle$ at different values of $\Gamma$ and temperature $\left(k_{\mathrm{B}} T=0.6,1.0\right)$ calculated within CRPA (solid lines) and exactly [60] (dash lines). Exact and approximate (CRPA) results for Ising model (short dash lines) coincide.

$2 \Gamma \cdot \operatorname{Re} \Psi^{z z}(0, E), k_{i}^{J}(0)$
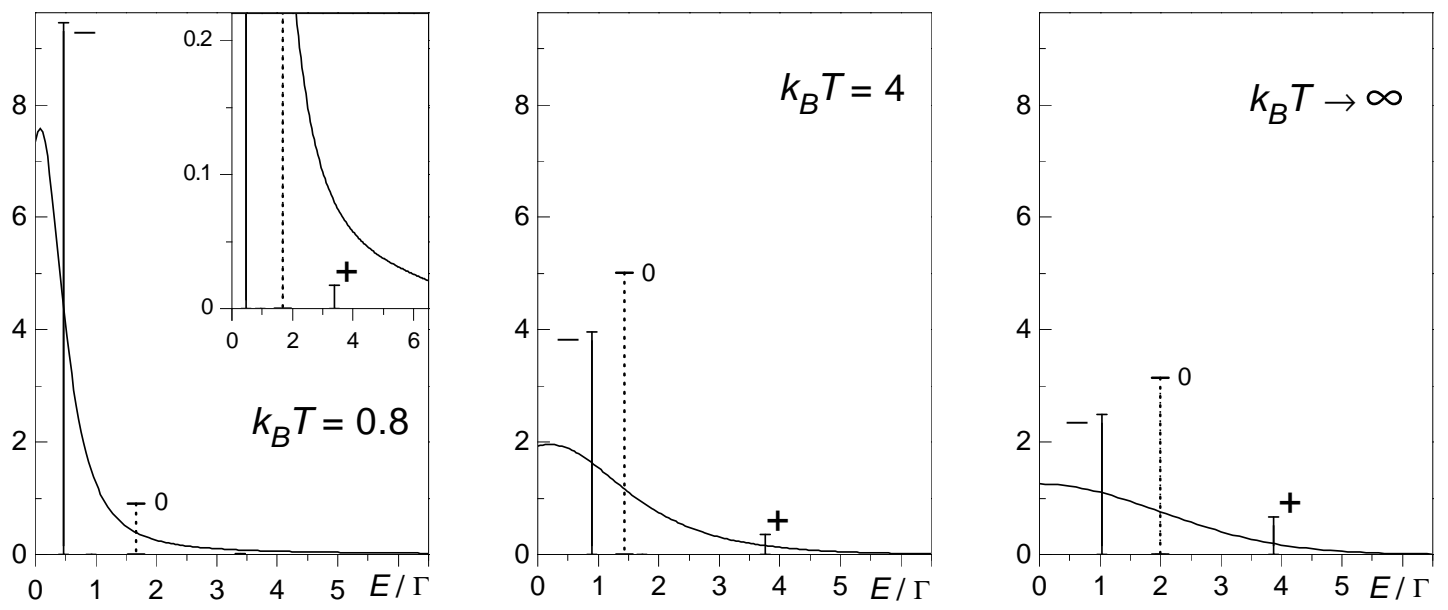

Figure 3. Frequency dependence of $2 \Gamma \cdot \operatorname{Re} \Psi^{z z}(0, E)$ for $\Gamma=1.0$ at different temperatures $\left(k_{\mathrm{B}} T=0.8,4, \infty\right)$ calculated numerically [35,36]. Vertical lines correspond to the mode integral intensities $k_{-}^{J}(0), k_{+}^{J}(0)$ within CRPA (solid line) and $k_{0}^{J}(0)$ within RPA (dash line). 

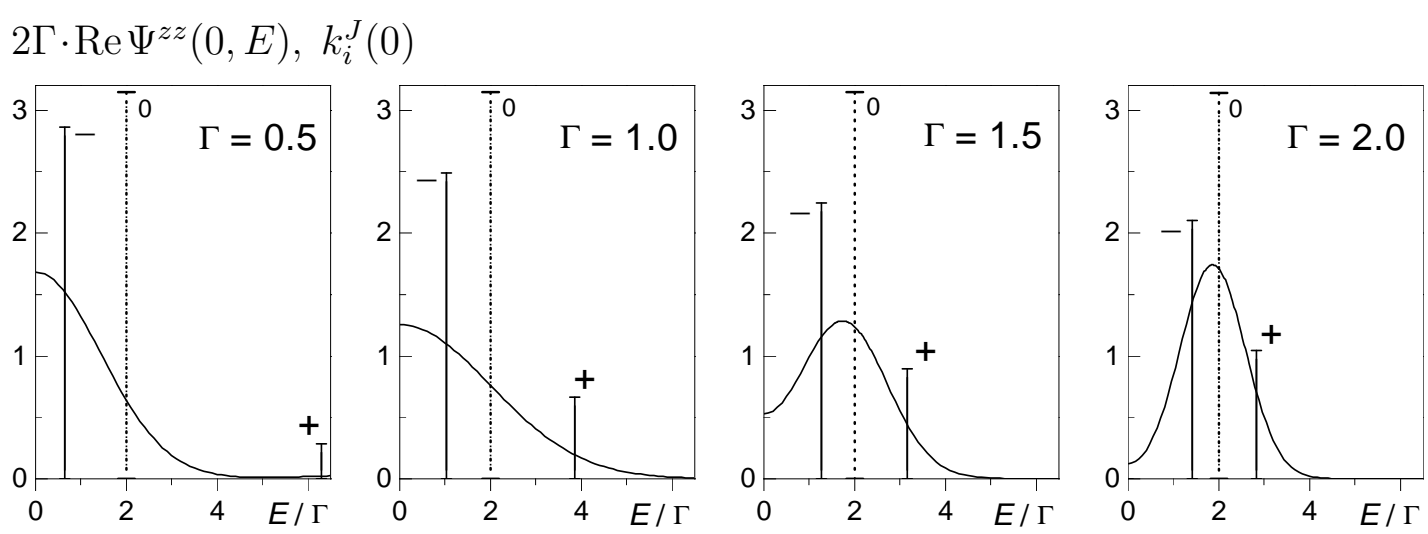

Figure 4. Exact results for frequency dependence of $2 \Gamma \cdot \operatorname{Re} \Psi^{z z}(0, E)$ at $T \rightarrow \infty$ at different values of $\Gamma(\Gamma=0.5,1.0,1.5,2.0)$. Vertical lines correspond to the mode integral intensities $k_{-}^{J}(0), k_{+}^{J}(0)$ within CRPA (solid line) and $k_{0}^{J}(0)$ within RPA (dash line).

$E_{-}(0)$ and $k_{+}^{J}(0)$. The fact that the resonance zone at $\Gamma=0.5$ is more prominent than at $\Gamma=1.0$ is described by decreasing $k_{-}^{J}(0)$ and increasing $k_{+}^{J}(0)$ at $\Gamma$ increasing. The fact that at $\Gamma=2.0$ the resonance zone $\operatorname{Re} \Psi^{z z}(0, E)$ is more prominent than at $\Gamma=$ 1.5 is described by closing in the positions of $E_{-}(0), E_{+}(0)$ modes at $\Gamma$ increasing. In figures 3, 4 we also depicted the results of the random phase approximation (RPA) for the short-range interactions. This approximation describes the frequency dependences of the real part of the relaxation function in the cases presented in figures 3, 4 qualitatively well only at $T \rightarrow \infty \Gamma=1.5,2.0$.

We restricted the presented numerical analysis of the longitudinal characteristics of the one-dimensional IMTF (at $J_{\nu \mu}=0$ ) by the values of the transverse field $0.2 \leqslant \Gamma \leqslant 2$. A more detailed analysis both at $J_{\nu \mu}=0$ and at $J_{\nu \mu} \neq 0$ and with the values of the microparameters corresponding to the $\mathrm{CsH}_{2} \mathrm{PO}_{4}$ crystal will be performed elsewhere.

\section{References}

1. Vaks V.G. Introduction to the Microscopic Theory of Ferroelectrics. Moskva, Nauka, 1973 (in Russian).

2. Blinc R., Žekš B. Soft Modes in Ferroelectrics and Antiferroelectrics. Amsterdam, North-Holland Publishing Company; New York, American Elsevier Publishing Company, 1974.

3. Lines M., Glass A. Principles and Applications of Ferroelectrics and Related Materials. Oxford, Clarendon Press, 1987.

4. Blinc R., Svetina S. // Phys. Rev., 1966, vol. 147, p. 423; vol. 147, p. 430.

5. Levitskii R.R., Korinevsky N.A., Stasyuk I.V. // Ukr. J. Phys., 1974, vol. 19, p. 1289 (in Russian).

6. Vaks V.G., Zein N.E., Strukov B.A. // Phys. Stat. Sol. (a), 1975, vol. 30, p. 801.

7. Vaks V.G., Zinenko V.I., Shneider V.E. // Usp. Fiz. Nauk, 1983, vol. 141, p. 620 (in Russian). 
8. Schmidt V.H. // Ferroelectrics, 1987, vol. 72, p. 157.

9. Blinc R., Žekš B. // Ferroelectrics, 1987, vol. 72, p. 193.

10. Havlin S. // Ferroelectrics, 1987, vol. 71, p. 183.

11. Levitsky R.R., Grigas J., Zachek I.R., Mits Ye.V., Paprotny W. // Ferroelectrics, 1986, vol. 67, p. 109.

12. Levitsky R.R., Zachek I.R., Kutny I.V., Schur J.J., Grigas J., Mizeris R. // Ferroelectrics, 1990, vol. 110, p. 85.

13. Steiner M., Villain J., Windsor C.G. // Adv. Phys., 1976, vol. 25, p. 87.

14. Izyumov Yu.A., Skriabin Yu.N. Statistical Mechanics of Magnetically Ordered System. New York, Consultants Bureau, 1988.

15. Yukhnovskii I.R. // Zhurn. Eksp. Teor. Fiz., 1958, vol. 34, p. 379 (in Russian).

16. Yukhnovskii I.R. On statistical theory of condensed system with long-range and shortrange interparticle interactions. Preprint of the Inst. Teor. Fiz., ITF-79-133R, Kiev, 1979 (in Russian).

17. Yukhnovskii I.R., Holovko M.F. Statistical Theory of Classical Equilibrium Systems, Kiev, Naukova Dumka, 1980 (in Russian).

18. Yukhnovskii I.R., Idzyk I.M., Kolomiets V.O. // Cond. Matt. Phys., 1993, No. 1, p. 5.

19. Yukhnovskii I.R. // Teor. Mat. Fiz., 1984, vol. 79, p. 282 (in Russian).

20. Patsahan O.V., Yukhnovskii I.R. // Teor. Mat. Fiz., 1990, vol. 83, p. 387 (in Russian).

21. Patsahan O.V. // Physica A, 1999, vol. 272, p. 358.

22. Yukhnovskii I.R. The method of displacements and collective variables. Preprint of the Inst. Teor. Fiz., ITF-71-26R, Kiev, 1971 (in Russian).

23. Vavrukh M.V. // Teor. Mat. Fiz., 1982, vol. 50, p. 438 (in Russian).

24. Vavrukh M.V. // Ukr. Fiz. Zhurn., 1991, vol. 36, p. 150 (in Russian).

25. Vavrukh M., Krokhmalskii T. // Phys. Stat. Sol. (b), 1991, vol. 168, p. 519; 1992, vol. 169, p. 451.

26. Yukhnovskii I.R., Levitskii R.R., Sorokov S.I. The theory of quasisipin system taking into account short-range interaction as a reference system. Expansion over inverse radius of long-range interaction. Preprint of the Inst. Teor. Fiz., ITF-86-132R, Kiev, 1986 (in Russian).

27. Yukhnovskii I.R., Sorokov S.I., Levitskii R.R. The theory of quasispin system taking into account short-range interaction as a reference system. Summation of reducible diagrams. Preprint of the Inst. Teor. Fiz., ITF-86-154R, Kiev, 1986 (in Russian).

28. Yukhnovskii I.R., Levitskii R.R., Sorokov S.I. Modern problems of statistical physics. - In: Proceedings of the All-Union Conference, vol. 1, Kiev, Naukova Dumka, 1989, p. 392 (in Russian).

29. Yukhnovskii I.R., Levitskii R.R., Sorokov S.I., Derzhko O.V. // Izv. AN SSSR, ser. fiz., 1991, vol. 55, p. 481 (in Russian).

30. Yukhnovskii I.R., Levitskii R.R., Sorokov S.I. // Cond. Matt. Phys., 1993, No. 1, p. 43 (in Ukrainian).

31. Kessel A.R., Berim G.O. Magnetic Resonance of Ising Magnets. Moskva, Nauka, 1982 (in Russian).

32. Zhelifonov M.P. // Teor. Mat. Fiz., 1971, vol. 8, p. 401 (in Russian).

33. Lieb E., Schultz T., Mattis D. // Ann. Phys., vol. 16, p. 407.

34. Tommet T.N., Huber D.L. // Phys. Rev. B., 1975, vol. 11, p. 450.

35. Derzhko O., Krokhmalskii T. // Phys. Rev. B., 1997, vol. 56, p. 11659.

36. Derzhko O.V., Krokhmalskii T. // Phys. Stat. Sol. (b), 1998, vol. 208, p. 221. 
37. Kubo R. // J. Phys. Soc. Japan, 1962, vol. 17, p. 110.

38. Smart J.S. Effective Field Theories of Magnetism. Philadelphia-London, W.B. Sauders Company, 1966.

39. Strieb B., Callen H.B. // Phys. Rev., 1963, vol. 130, p. 1798.

40. Vaks V.G., Zein N.E. // Zhurn. Eksp. Teor. Fiz., 1974, vol. 67, p. 1082 (in Russian).

41. Vaks V.G., Zein N.E. // Solid State Phys., 1975, vol. 17, p. 1617 (in Russian).

42. Dzyub I.P. // Phys. Stat. Sol. (b), 1974, vol. 61, p. 383.

43. Saxena V.K. // Phys. Rev. B., 1983, vol. 27, p. 6884.

44. Levitskii R.R., Korinevsky N.A., Stasyuk I.V. // Phys. Stat. Sol. (b), 1978, vol. 88, p. 51.

45. Levitskii R.R., Zachek I.P., Varanitskii V.I. Distribution functions and relaxation processes of the Ising model. Cluster approximation. Preprint of the Inst. Teor. Fiz., ITF-78-41R, Kiev, 1978 (in Russian).

46. Havlin S., Sompolinsky H. // Phys. Rev. B., 1982, vol. 25, p. 5828.

47. Yukhnovskii I.R., Levitskii R.R., Sorokov S.I. Thermodynamic and distribution functions for Ising model. Two-particle cluster approximation. Preprint of the Inst. Teor. Fiz., ITF-86-142R, Kiev, 1986 (in Russian).

48. Levitskii R.R., Sorokov S.I. // Cond. Matt. Phys., 1994, No. 3, p. 79.

49. Moina A.P. // Cond. Matt. Phys., 1997, No. 10, p. 93.

50. Stasyuk I.V., Levitskii R.R., Korinevsky N.A. // Phys. Stat. Sol. (b), 1979, vol. 91, p. 541.

51. Levitskii R.R., Stasyuk I.V., Korinevsky N.A. // Ferroelectrics, 1978, vol. 21, p. 481.

52. Korinevsky N.A., Levitskii R.R. // Teor. Mat. Fiz., 1980, vol. 42, p. 416 (in Russian).

53. Blinc R., Schmidth V.H. // Ferroelectrics Letters, 1984, vol. 1, p. 119.

54. Levitskii R.R. Sorokov S.I. Theory of quasi-spin system with short-range interactions taken into account (in therms of the basis) in the two-particle cluster approximation. Application to Heisenberg model. Preprint of the Inst. Teor. Fiz., ITF-87-28R, Kiev, 1987 (in Russian).

55. Sorokov S.I., Levitskii R.R., Baran O.R. The study of Ising model within cluster expansion method. Preprint of the Inst. Fiz. Kond. System., IFKS-92-18U, Lviv, 1992 (in Ukrainian).

56. Sorokov S.I., Levitskii R.R., Baran O.R. // Ukr. J. Phys., 1996, vol. 41, p. 490 (in Ukrainian).

57. Slobodyan P.M., Stasyuk I.V. Teor. Mat. Fiz., 1974, vol. 19, p. 423 (in Russian).

58. Levitskii R.R. Sorokov S.I. Dynamics and thermodynamic properties of the Ising model in a transverse field. The two-particle cluster approximation. Preprint of the Inst. Teor. Fiz., ITF-88-34R, Kiev, 1988 (in Russian).

59. Izyumov Yu.A., Kassan-Ogly F.A., Skriabin Yu.N. Field Method in Theory of Ferromagnetism. Moskva, Nauka, 1974 (in Russian).

60. Pfeuty P. // Ann. Phys., 1970, vol. 57, p. 79. 


\title{
Базисний підхід в теорії псевдоспінових систем
}

\author{
Р.Р.Левицький, С.І.Сороков, О.Р.Баран
}

Інститут фізики конденсованих систем НАН України, 79011 Львів, вул. Свєнціцького, 1

\section{Отримано 18 квітня 2000 р.}

Для теоретичного опису псевдоспінових систем з суттєвими короткосяжними та далекосяжними взаємодіями використовується метод, який грунтується на розрахунку функціоналу вільної енергії 3 базисним урахуванням короткосяжних взаємодій у кластерному наближенні. Для квантових псевдоспінових систем запропоновано послідовне формулювання методу кластерних розвинень та метод, який дає змогу в рамках кластерного наближення отримати для базисних температурних кумулянтних функцій Гріна довільного порядку рівняння типу рівнянь Орнштейна-Церніке. В наближенні двочастинкового кластера в явному вигляді отримано вираз для парної температурної кумулянтної функції Гріна базисної системи. У кластерному наближенні хаотичних фаз розраховані та досліджені термодинамічні характеристики, спектр елементарних збуджень та інтегральні інтенсивності гілок спектра моделі Ізінга в поперечному полі.

Ключові слова: фазові переходи, псевдоспінові моделі, базисний підхід, кластерне наближення, рівняння Орнштейна-Церніке, м'яка мода

PACS: 03.65. $-w, 05.30 .-d$ 
